(C) 2016 IEEE. Personal use of this material is permitted. Permission from IEEE must be obtained for all other uses, in any current or future media, including reprinting/republishing this material for advertising or promotional purposes, creating new collective works, for resale or redistribution to servers or lists, or reuse of any copyrighted component of this work in other works. 


\title{
Zero-knowledge proof systems for QMA
}

\author{
Anne Broadbent ${ }^{1} \quad$ Zhengfeng Ji $^{2,3} \quad$ Fang Song $^{4} \quad$ John Watrous $^{5,6}$ \\ ${ }^{1}$ Department of Mathematics and Statistics \\ University of Ottawa, Canada \\ ${ }^{2}$ Centre for Quantum Computation and Intelligent Systems, School of Software \\ Faculty of Engineering and Information Technology \\ University of Technology Sydney, Australia \\ ${ }^{3}$ State Key Laboratory of Computer Science, Institute of Software \\ Chinese Academy of Sciences, China \\ ${ }^{4}$ Institute for Quantum Computing and Department of Combinatorics E Optimization \\ University of Waterloo, Canada \\ ${ }^{5}$ Institute for Quantum Computing and School of Computer Science \\ University of Waterloo, Canada \\ ${ }^{6}$ Canadian Institute for Advanced Research \\ Toronto, Canada
}

September 29, 2018

\begin{abstract}
Prior work has established that all problems in NP admit classical zero-knowledge proof systems, and under reasonable hardness assumptions for quantum computations, these proof systems can be made secure against quantum attacks. We prove a result representing a further quantum generalization of this fact, which is that every problem in the complexity class QMA has a quantum zero-knowledge proof system. More specifically, assuming the existence of an unconditionally binding and quantum computationally concealing commitment scheme, we prove that every problem in the complexity class QMA has a quantum interactive proof system that is zero-knowledge with respect to efficient quantum computations.

Our QMA proof system is sound against arbitrary quantum provers, but only requires an honest prover to perform polynomial-time quantum computations, provided that it holds a quantum witness for a given instance of the QMA problem under consideration. The proof system relies on a new variant of the QMA-complete local Hamiltonian problem in which the local terms are described by Clifford operations and standard basis measurements. We believe that the QMA-completeness of this problem may have other uses in quantum complexity.
\end{abstract}




\section{Introduction}

Zero-knowledge proof systems, first introduced by Goldwasser, Micali and Rackoff [23], are interactive protocols that allow a prover to convince a verifier of the validity of a statement while revealing no additional information beyond the statement's validity. Although paradoxical as it appears, several problems that are not known to be efficiently computable, such as the Quadratic Non-Residuosity, Graph Isomorphism, and Graph Non-Isomorphism problems, were shown to admit zero-knowledge proof systems [21,23]. Under reasonable intractability assumptions, Goldreich, Micali and Wigderson [21] gave a zero-knowledge protocol for the Graph 3-Coloring problem and, because of its NP-completeness, for all NP problems. This line of work was further extended in [7], which showed that all problems in IP have zero-knowledge proof systems.

Since the invention of this concept, zero-knowledge proof systems have become a cornerstone of modern theoretical cryptography. In addition to the conceptual innovation of formulating a complexity-theoretic notion of knowledge, zero-knowledge proof systems are essential building blocks in a host of cryptographic constructions. One notable example is the design of secure twoparty and multi-party computation protocols [20].

The extensive works on zero-knowledge largely reside in a classical world. The development of quantum information science and technology has urged another look at the landscape of zeroknowledge proof systems in a quantum world. Namely, both honest users and adversaries may potentially possess the capability to exchange and process quantum information. There are, of course, zero-knowledge protocols that immediately become insecure in the presence of quantum attacks due to efficient quantum algorithms that break the intractability assumptions upon which these protocols rely. For instance, Shor's quantum algorithms for factoring and computing discrete logarithms [42] invalidate the use of these problems, generally conjectured to be classically hard, as a basis for the security of zero-knowledge protocols against quantum attacks. Even with computational assumptions against quantum adversaries, however, it is still highly nontrivial to establish the security of classical zero-knowledge proof systems in the presence of malicious quantum verifiers because of a technical reason that we now briefly explain.

The zero-knowledge property of a proof system for a fixed input string is concerned with the computations that may be realized through an interaction between a (possibly malicious) verifier and the prover. That is, the malicious verifier may take an arbitrary input (usually called the auxiliary input to distinguish it from the input string to the proof system under consideration), interact with the prover in any way it sees fit, and produce an output that is representative of what it has learned through the interaction. Roughly speaking, the prover is said to be zero-knowledge on the fixed input string if any computation of the sort just described can be efficiently approximated ${ }^{1}$ by a simulator operating entirely on its own-meaning that it does not interact with the prover, and in the case of an NP problem it does not possess a witness for the fixed problem instance being considered. The proof system is then said to be zero-knowledge when this zero-knowledge property holds for all yes-instances of the problem under consideration.

Classically speaking, the zero-knowledge property is typically established through a technique known as rewinding. In essence, the simulator can store a copy of its auxiliary input, and it can make guesses and store intermediate states representing a hypothetical prover/verifier interaction - and if it makes a bad guess or otherwise experiences bad luck when simulating this hypothetical interaction, it simply reverts to an earlier stage (or possibly back to the beginning)

\footnotetext{
${ }^{1}$ Different notions of approximations are considered, including statistical approximations and computational approximations, which require that the simulator's computation is either statistically (or information-theoretically) indistinguishable or computationally indistinguishable from the malicious verifier's computation. This paper is primarily concerned with the computational variant.
} 
of the simulation and tries again. Indeed, it is generally the simulator's freedom to disregard the temporal restrictions of the actual prover/verifier interaction in a way such as this that makes it possible to succeed.

However, rewinding a quantum simulation is more problematic; the no-cloning theorem [51] forbids one from copying quantum information, making it impossible to store a copy of the input or of an intermediate state, and measurements generally have an irreversible effect [16] that may partially destroy quantum information. Such difficulties were first observed by van de Graaf [45] and further studied in $[11,46]$. Later, a quantum rewinding technique was found [49] to establish that several interactive proof systems, including the Goldreich-Micali-Wigderson Graph 3-Coloring proof system [21], remain zero-knowledge against malicious quantum verifiers (under appropriate quantum intractability assumptions in some cases). It follows that all NP problems have zero-knowledge proof systems even against quantum malicious verifiers, provided that a quantum analogue of the intractability assumption required by the Goldreich-Micali-Wigderson Graph 3-Coloring proof system are in place.

This work studies the quantum analogue of NP, known as QMA, in the context of zeroknowledge. These are problems with a succinct quantum witness satisfying similar completeness and soundness to NP (or its randomized variant MA). Quantum witnesses and verification are conjectured to be more powerful than their classical counterparts: there are problems that admit short quantum witnesses, whereas there is no known method for verification using a polynomialsized classical witness. In other words, NP $\subseteq$ QMA holds trivially, and the containment is typically conjectured to be proper. The question we address in this paper is: Does every problem in QMA have a zero-knowledge quantum interactive proof system? In more philosophical terms, viewing quantum witnesses as precious sources of knowledge: Can one always devise a proof system that reveals nothing about a quantum witness beyond its validity?

\subsection{Our contributions}

We answer the above question positively by constructing a quantum interactive proof system for any problem in QMA that is zero-knowledge against any polynomial-time quantum adversary, under a reasonable quantum intractability assumption.

Theorem 1. Assuming the existence of an unconditionally binding and quantum computationally concealing bit commitment scheme, every problem in QMA has a quantum computational zero-knowledge proof system.

A few of the desirable features of our proof system are as follows:

1. Our proof system has a simple structure, similar to the classical Goldreich-Micali-Wigderson Graph 3-Coloring proof system (and to the so-called $\Sigma$-protocols more generally). It can be viewed as a three-phase process: the prover commits to a quantum witness, the verifier makes a random challenge, and finally the prover responds to the challenge by partial opening of the committed information that suffices to certify the validity.

2. All communications in our proof system are classical except for the first commitment message, and the verifier can measure the quantum message immediately upon its arrival (which has a strong technological appeal).

3. Our protocol is based on mild computational assumptions. The sort of bit commitment scheme it requires can be implemented, for instance, under the existence of injective one-way functions that are hard to invert in quantum polynomial time. 
4. Our protocol is prover-efficient. It is sound against general quantum provers, but given a valid quantum witness, an honest prover only needs to perform efficient quantum computations. As has already been suggested, aside from the preparation of the first quantum message, all of the remaining computations performed by the honest prover are classical polynomial-time computations.

As a key ingredient of our zero-knowledge proof system, we introduce a new variant of the $k$-local Hamiltonian problem and prove that it remains QMA-complete (with respect to Karp reductions). The $k$-local Hamiltonian problem asks if the minimum eigenvalue (or ground state energy in physics parlance) of an $n$-qubit Hamiltonian $H=\sum_{j} H_{j}$, where each $H_{j}$ is $k$-local (i.e., acts trivially on all but $k$ of the $n$ qubits), is below a particular threshold value. This problem was introduced and proved to be QMA-complete (for the case $k=5$ ) by Kitaev [34]. We show that each $H_{j}$ can be restricted to be realized by a Clifford operation, followed by a standard basis measurement, and the QMA-completeness is preserved. Beyond its use in this paper, this fact has the potential to provide other insights into the study of quantum Hamiltonian complexity. For an arbitrary problem $A \in \mathrm{QMA}$, we can reduce an instance of $A$ efficiently to an instance of the $k$-local Clifford Hamiltonian problem, and a valid witness for $A$ can also be transformed into a witness for the corresponding $k$-local Clifford Hamiltonian problem instance by an efficient quantum procedure. As a result, $A$ has a zero-knowledge proof system by composing this reduction with our zero-knowledge proof system for the $k$-local Clifford Hamiltonian problem.

Our proof system also employs a new encoding scheme for quantum states, which we construct by extending the trap scheme proposed in [10]. While our new scheme can be seen as a quantum authentication scheme (cf. $[2,5,6]$ ), it in addition allows performing arbitrary constantqubit Clifford circuits and measuring in the computational basis directly on authenticated data without the need for auxiliary states. Previously the only known scheme supporting this feature requires high-dimensional quantum systems (i.e., qudits rather than qubits) [6], which make it inconvenient in our setting where all quantum operations are on qubits.

\subsection{Overview of protocol and techniques}

A natural approach to constructing zero-knowledge proofs for QMA is to consider a quantum analogue of the Goldreich-Micali-Wigderson proof system for Graph 3-Coloring (which we will hereafter refer to as the GMW 3-Coloring proof system). Let us focus in particular on the local Hamiltonian problem, and consider a proof system in which the prover holds a quantum witness state for an instance of this problem, commits to this witness, and receives the challenge from the verifier (which, let us say, is a random term of the local Hamiltonian). The prover might then open the commitments of the set of qubits on which the term acts non-trivially so that the verifier can measure the local energy for this term and determine acceptance accordingly.

There is a major difficulty when one attempts to carry out such an approach for QMA. The zero-knowledge property of the GMW 3-Coloring proof system depends crucially on a structural property of the problem: the honest prover is free to randomize the three colors used in its coloring, and when the commitments to the colors of two neighboring vertices are revealed, the verifier will see just a uniform mixture over all pairs of different colors. This uniformity of the coloring marginals is important in achieving the zero-knowledge property of the proof system. Unlike the case of 3-Coloring, however, none of the known QMA-complete problems under Karp reductions has such desirable properties. For example, if we use local Hamiltonian problems directly in a GMW-type proof system, of the sort suggested above, information about the reduced state of the quantum witness will be leaked to the verifier, possibly violating the zero-knowledge requirement. 
To overcome the difficulty suggested above, we employ several ideas that enable the prover to "partially" open the commitments, revealing only the fact that the committed state lives in certain subspaces, and nothing further. Our first technique simplifies the verification circuit for QMAcomplete problems through the introduction of the local Clifford-Hamiltonian problem that was already described. Somewhat more specifically, our formulation of this problem requires every Hamiltonian term to take the form $C^{*}\left|0^{k}\right\rangle\left\langle 0^{k}\right| C$ for some Clifford operation $C$. Because the local Clifford-Hamiltonian problem remains QMA-complete, it implies a random Clifford verification procedure for problems in QMA: intuitively, the verification of a quantum witness has been simplified to a Clifford measurement followed by a classical verification.

The Clifford verification procedure works in harmony with the encryption of quantum data via the quantum one-time pad and other derived hybrid schemes that are used by our proof system. This has the important effect of transforming statements about quantum states into those about the classical keys of the quantum one-time pad, which naturally leads to our second main idea: the use of zero-knowledge proofs for NP against quantum attacks to simplify the construction of zero-knowledge proofs for QMA. In our protocol, the verifier measures the encrypted quantum data and asks the prover to prove, using a zero-knowledge protocol for $\mathrm{NP}$, that the decryption of this result is consistent with the verifier accepting.

In fact, if the verifier measures the quantum data according to the specifications of the protocol, the combination of the Clifford verification and the use of zero-knowledge proofs for NP suffices. A problem arises, however, if the verifier does not perform the honest measurement. Our third technique, inspired by work on quantum authentication $[2,6,10,14]$, employs a new scheme for encoding quantum states. Roughly speaking, if the prover encodes a witness state under our encoding scheme, then the verifier is essentially forced to perform the measurement honestly-any attempt to fake a "logically different" measurement result will succeed with negligible probability. In our proof system, we adapt the trap scheme proposed in [10] so that we can perform any constant-sized Clifford operations on authenticated quantum data followed by computational basis measurements, benefiting along the way from ideas concerning quantum computation on authenticated quantum data.

The resulting zero-knowledge proof system for QMA has a similar overall structure to the GMW 3-Coloring protocol: the prover encodes the quantum witness state using a quantum authentication scheme, and sends the encoded quantum data together with a commitment to the secret keys of the authentication to the verifier. The verifier randomly samples a term $C^{*}\left|0^{k}\right\rangle\left\langle 0^{k}\right| C$ in the local Clifford-Hamiltonian problem, applies the operation $C$ transversally on the encoded quantum data and measures all qubits corresponding to the $k$ qubits of the selected term in the computational basis, and sends the measurement outcomes to the prover. The prover and verifier then invoke a quantum-secure zero-knowledge proof for the NP statement that the commitment correctly encodes an authentication key and, under this key, the verifier's measurement outcomes do not decode to $0^{k}$.

\subsection{Comparisons to related work}

There has been other work on quantum complexity and theoretical cryptography, some of which is discussed below, that allows one to conclude statements having some similarity to our results. We will argue, however, that with respect to the problem of devising zero-knowledge quantum interactive proof systems for QMA, our main result is stronger in almost all respects. In addition, we believe that our proof system is appealing both because it is conceptually simple and represents a natural extension of well-known classical methods. 
1. Zero-knowledge proof systems for all of IP. Hallgren, Kolla, Sen and Zhang [27] proved that classical zero-knowledge proof systems for IP [7] can be made secure against malicious quantum verifiers under a certain technical condition. It appears that this condition holds assuming the existence of a quantum computationally hiding commitment scheme. Because QMA is contained in IP, this would imply a classical zero-knowledge protocol for QMA. However, this generic protocol would require a computationally unbounded prover to carry out the honest protocol, and it is unlikely to reduce the round complexity without causing unexpected consequences in complexity theory $[22,24,47]$.

2. Secure two-party computations. Another approach to constructing zero-knowledge proofs for QMA is to apply the general tool of secure two-party quantum computation $[6,13,14]$. In particular, we may imagine two parties, a prover and a verifier, jointly evaluating the verification circuit of a QMA problem, with the prover holding a quantum witness as his/her private input. In principle, one can design a two-party computation protocol so that the verifier learns the validity of the statement but nothing more about the prover's private input. While we believe that a careful analysis could make this approach work, it comes at a steep cost. First, we need to make significantly stronger computational assumptions, as secure quantum two-party computation relies on (at least) secure computations of classical functions against quantum adversaries. The best-known quantum-secure protocols for classical two-party computation assume quantum-secure dense public-key encryption [28] or similar primitives [36], in contrast to the existence of a quantum computationally hiding commitment scheme. ${ }^{2}$ Secondly, the protocol obtained this way is only an argument system. That is, the protocol is only sound against computationally bounded dishonest provers. Moreover, the generic quantum two-party computation protocol evaluates the verification circuit gate by gate, and in particular interactions are unavoidable for some (non-Clifford) gates. This causes the round complexity to grow in proportion to the size of the verification circuit. In addition, the communications are inherently quantum, which makes the protocol much more demanding from a technological viewpoint.

On the positive side, through this approach, it is possible to achieve negligible soundness error using just one copy of witness state. In contrast, our proof system directly inherits the soundness error of the most natural and direct verification for the local Clifford-Hamiltonian problem (i.e., randomly select a Hamiltonian term and measure). If one reduces an arbitrary QMA-verification procedure to an instance of this problem, the resulting soundness guarantee could be significantly worse.

3. Zero-knowledge proofs for Density Matrix Consistency. It was pointed out by Liu [35] that the Density Matrix Consistency problem, which asks if there exists a global state of $n$ qubits that is consistent with a collection of $k$-qubit density matrix marginals, should admit a simple zeroknowledge proof system following the GMW 3-Coloring approach. This fact was one of the inspirations for our work. While it approaches our main result, it does not necessarily admit a zero-knowledge proof system for all problems in QMA, as the Density Matrix Consistency problem is only known to be hard for QMA with respect to Cook reductions.

4. Other results on Clifford verifications for QMA. We note that Clifford verification with classical post-processing of QMA was considered in [38] using magic states as ancillary resources. Our construction is arguably simpler, uses only constant-size Clifford operations, and most importantly does not require any resource states. This helps to avoid checking the correctness of resource states in the final zero-knowledge protocol. We are hopeful that our techniques will

\footnotetext{
2 Roughly speaking, this distinction is analogous to "Cryptomania" vs "minicrypt" according to Impagliazzo's fiveworld paradigm [30].
} 
provide new insights to the study of quantum Hamiltonian complexity, and may find useful applications in other areas of research such as the study of non-local games. One byproduct of our Clifford-Hamiltonian reduction proof is an alternative proof of the single-qubit measurement verification for QMA recently proposed by [39].

\section{Organization}

The remainder of the paper is organized as follows. Section 2 describes the variant of the local Hamiltonian problem mentioned above. We present our zero-knowledge proof system for QMA in Section 3 and prove its completeness and soundness in Section 4 and zero-knowledge property in Section 5. We conclude with some remarks and future directions in Section 6. An appendix summarizing basic notation, definitions, and useful primitives for the construction of our zeroknowledge proof system is also included for completeness.

\section{The local Clifford-Hamiltonian problem}

The local Hamiltonian problem [34] is a well-known example of a complete problem for QMA, provided that certain assumptions are in place regarding the gap between the ground state energy (i.e., the smallest eigenvalue) of input Hamiltonians for yes- and no-inputs. A general and somewhat imprecise formulation of the local Hamiltonian problem is as follows.

The $k$-local Hamiltonian problem $(k-L H)$

Input: A collection $H_{1}, \ldots, H_{m}$ of $k$-local Hamiltonian operators, each acting on $n$ qubits and satisfying $0 \leq H_{j} \leq \mathbb{1}$ for $j=1, \ldots, m$, along with real numbers $\alpha$ and $\beta$ satisfying $\alpha<\beta$.

Yes: $\quad$ There exists an $n$-qubit state $\rho$ such that $\left\langle\rho, H_{1}+\cdots+H_{m}\right\rangle \leq \alpha$.

No: $\quad$ For every $n$-qubit state $\rho$, it holds that $\left\langle\rho, H_{1}+\cdots+H_{m}\right\rangle \geq \beta$.

This problem statement is imprecise in the sense that it does not specify how $\alpha$ and $\beta$ are to be represented or what requirements are placed on the gap $\beta-\alpha$ mentioned above. We will be more precise about these issues when formulating a restricted version of this problem below, but it is appropriate that we first summarize what is already known.

It is known that $k$-LH is complete for QMA (with respect to Karp reductions) provided $\alpha$ and $\beta$ are input in a reasonable way and separated by an inverse polynomial gap; this was first proved by Kitaev [34] for the case $k=5$, then by Kempe and Regev [32] for $k=3$ and Kempe, Kitaev, and Regev [31] for $k=2$. If one adds the additional requirement that $\alpha$ is exponentially small, which will be important in the context of this paper, then QMA-completeness for $k=5$ still follows from Kitaev's proof, but the proofs of Kempe and Regev and Kempe, Kitaev, and Regev do not imply the same for $k=3$ and $k=2$. On the other hand, the work of Bravyi [9] and Gosset and Nagaj [25] does establish QMA-completeness for exponentially small $\alpha$, for $k=4$ and $k=3$, respectively.

The restricted version of the local Hamiltonian we introduce is one in which each Hamiltonian term $H_{j}$ is not only $k$-local and satisfies $0 \leq H_{j} \leq \mathbb{1}$, but furthermore on the $k$ qubits on which it acts nontrivially, its action must be given by a rank 1 projection operator of the form

$$
C_{j}^{*}\left|0^{k}\right\rangle\left\langle 0^{k}\right| C_{j},
$$

for some choice of a $k$-qubit Clifford operation $C_{j}$. For brevity, we will refer to any such operator as a $k$-local Clifford-Hamiltonian projection. The precise statement of our problem variant is as follows. 
The k-local Clifford-Hamiltonian problem $(k-L C H)$

Input: A collection $H_{1}, \ldots, H_{m}$ of $k$-local Clifford-Hamiltonian projections, along with positive integers $p$ and $q$ expressed in unary notation (i.e., as strings $1^{p}$ and $1^{q}$ ) and satisfying $2^{p}>q$.

Yes: $\quad$ There exists an $n$-qubit state $\rho$ such that $\left\langle\rho, H_{1}+\cdots+H_{m}\right\rangle \leq 2^{-p}$.

No: $\quad$ For every $n$-qubit state $\rho$, it holds that $\left\langle\rho, H_{1}+\cdots+H_{m}\right\rangle \geq 1 / q$.

It may be noted that, by the particular way we have stated this problem, we are focusing on a variant of the local Hamiltonian problem in which the parameter $\alpha$ may be exponentially small and the gap $\beta-\alpha$ is at least inverse polynomial.

Theorem 2. The 5-local Clifford-Hamiltonian problem is QMA-complete with respect to Karp reductions. Moreover, for any choice of promise problem $A=\left(A_{\text {yes }}, A_{n o}\right) \in$ QMA and a polynomially bounded function $p$, there exists a Karp reduction $f$ from $A$ to 5-LCH having the form

$$
f(x)=\left\langle H_{1}, \ldots, H_{m}, 1^{p(|x|)}, 1^{q}\right\rangle
$$

for every $x \in A_{\text {yes }} \cup A_{\text {no }}$.

Proof. The containment of the 5-local Clifford-Hamiltonian problem in QMA follows from the fact that the 5-LH problem is in QMA for the same choice of the ground state energy bounds. It therefore remains to prove the statement concerning the QMA-hardness of the 5-LCH problem.

Let $A=\left(A_{\mathrm{yes}}, A_{\mathrm{no}}\right)$ be any promise problem in QMA and let $p$ be a polynomially bounded function. Using a standard error reduction procedure for QMA, one may conclude that there exists a polynomial-time generated collection $\left\{V_{x}: x \in A_{\text {yes }} \cup A_{\text {no }}\right\}$ of measurement circuits having these properties:

1. If $x \in A_{\text {yes, }}$ there exists a state $\rho$ such that $V_{x}(\rho)=1$ with probability $1-2^{-p(|x|)}$.

2. If $x \in A_{\text {no, }}$, then for all quantum states $\rho$ representing valid inputs to $V_{x}$ it holds that $V_{x}(\rho)=1$ with probability at most $1 / 2$.

It is known that $\{\Lambda(P), H\}$ is a universal gate set for quantum computation, so there would be no loss of generality in assuming each $V_{x}$ is a quantum circuit using gates from this set, together with a supply of ancillary qubits initialized to the state $|0\rangle$. For technical reasons (which are discussed later) we will assume something marginally stronger, which is that each $V_{x}$ uses gates from the set $\{\Lambda(P), H \otimes H\}$. That is, every Hadamard gate appearing in $V_{x}$ is paired with another Hadamard gate to be applied at the same time but on a different qubit. Note that for any circuit composed of gates from the set $\{\Lambda(P), H\}$, this stronger condition is easily met by adding to this circuit a number of additional Hadamard gates on an otherwise unused ancilla qubit.

Now consider the 5-local circuit-to-Hamiltonian construction of Kitaev [34], for a given choice of $V_{x}$. In this construction, the resulting Hamiltonians have the form

$$
H_{\text {total }}=H_{\text {in }}+H_{\text {out }}+H_{\text {clock }}+H_{\text {prop }},
$$

where the terms check the initialization, readout, validity of unary clock, and propagation of computation respectively. It follows from Kitaev's proof that, for $x \in A_{\text {yes, }}$, the resulting Hamiltonian $H_{\text {total }}$ has ground state energy at most $2^{-p(|x|)}$, and for $x \in A_{\text {no }}$ the ground state energy of $H_{\text {total }}$ is at least $1 / q(|x|)$, for some polynomially bounded function $q$. To complete the proof, it suffices to 
demonstrate that each of these terms can be expressed as a sum of Clifford-Hamiltonian projections.

The first three terms, $H_{\text {in }}, H_{\text {out }}$, and $H_{\text {clock }}$, can be expressed as sums of Clifford-Hamiltonian projections easily, as they are all projection operators that are diagonal in the standard basis. The propagation term has the form $H_{\text {prop }}=\sum_{t=1}^{T} H_{\text {prop }, t}$ where each operator $H_{\text {prop }, t}$ takes the form

$$
\begin{aligned}
H_{\text {prop }, t}= & \frac{1}{2}\left[\left(|100\rangle\left\langle\left. 100\right|_{t-1, t, t+1}+\mid 110\right\rangle\left\langle\left. 110\right|_{t-1, t, t+1}\right) \otimes \mathbb{1}\right.\right. \\
& -|110\rangle\left\langle\left. 100\right|_{t-1, t, t+1} \otimes U_{t}-\mid 100\right\rangle\left\langle\left. 110\right|_{t-1, t, t+1} \otimes U_{t}^{*}\right] \\
= & |10\rangle\left\langle1 0 | _ { t - 1 , t + 1 } \otimes \frac { 1 } { 2 } \left[\mathbb{1}_{t} \otimes \mathbb{1}-|1\rangle\left\langle\left. 0\right|_{t} \otimes U_{t}-\mid 0\right\rangle\left\langle\left. 1\right|_{t} \otimes U_{t}^{*}\right] .\right.\right.
\end{aligned}
$$

Here, the first three qubits (indexed by $t-1, t$, and $t+1$ ) refer to qubits in a clock register and $U_{t}$ represents the $t$-th unitary gate in $V_{x}$. To prove that each propagation operator $H_{\text {prop, } t}$ can be expressed as a sum of Clifford-Hamiltonian projections, it suffices to prove the same for every projection of the form

$$
\frac{1}{2}\left[\mathbb{1} \otimes \mathbb{1}-|1\rangle\langle 0|\otimes U-| 0\rangle\langle 1| \otimes U^{*}\right],
$$

for $U$ being either $\Lambda(P)$ or $H \otimes H$.

In the case that $U=\Lambda(P)$, one has that the projection (5) is the sum of the four CliffordHamiltonian projections corresponding to these vectors:

$$
\begin{aligned}
& |-\rangle|00\rangle=(Z H \otimes \mathbb{1} \otimes \mathbb{1})|000\rangle, \\
& |-\rangle|01\rangle=(Z H \otimes \mathbb{1} \otimes X)|000\rangle, \\
& |-\rangle|10\rangle=(Z H \otimes X \otimes \mathbb{1})|000\rangle, \\
& |\circlearrowright\rangle|11\rangle=\left(P^{*} H \otimes X \otimes X\right)|000\rangle,
\end{aligned}
$$

where $|\circlearrowright\rangle=(|0\rangle-i|1\rangle) / \sqrt{2}$. In the case that $U=H \otimes H$, one has that the projection (5) is the sum of the four Clifford-Hamiltonian projections corresponding to these vectors:

$$
\begin{aligned}
& \left|\psi_{1}\right\rangle=(|000\rangle-|011\rangle-|101\rangle-|110\rangle) / 2, \\
& \left|\psi_{2}\right\rangle=(|000\rangle+|011\rangle-|100\rangle-|111\rangle) / 2, \\
& \left|\psi_{3}\right\rangle=(|001\rangle-|010\rangle+|101\rangle-|110\rangle) / 2, \\
& \left|\psi_{4}\right\rangle=(|001\rangle+|010\rangle-|100\rangle+|111\rangle) / 2 .
\end{aligned}
$$

All four of these vectors are obtained by a Clifford operation applied to the all-zero state. In particular, when the following Clifford circuits are applied to the state $|000\rangle$, the states $\left|\psi_{1}\right\rangle,\left|\psi_{2}\right\rangle,\left|\psi_{3}\right\rangle$, and $\left|\psi_{4}\right\rangle$ are obtained:
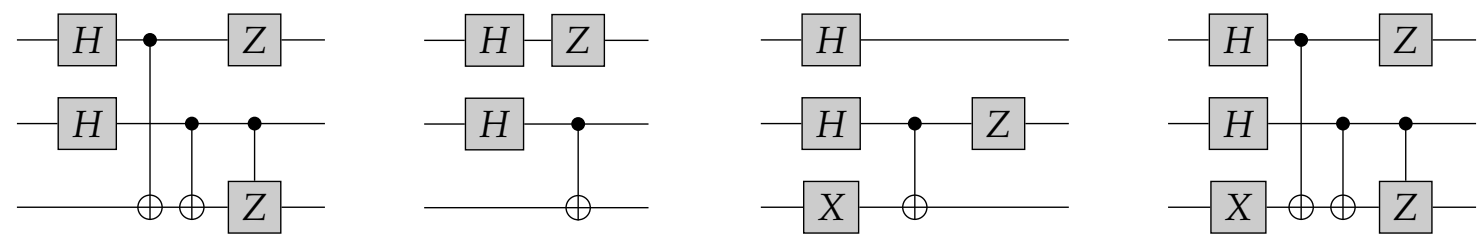

This completes the proof.

Remark 1. If one is given a witness to a given QMA problem $A$, it is possible to efficiently compute a witness to the corresponding $k$-local Hamiltonian problem instance through Kitaev's reduction. Our reduction also inherits this property. 
Remark 2. There is no loss of generality in setting $q=1$ in the statement of the $k$-LCH problem, meaning that Theorem 2 holds for this somewhat simplified problem statement. This may be proved by repeating each Hamiltonian term $q$ times in a given problem instance and adjusting $p$ as necessary.

Remark 3. States of the form $C\left|0^{k}\right\rangle$, for a Clifford operation $C$, are stabilizer states of $k$ qubits. Theorem 2 therefore implies that there exists a QMA verification procedure in which the verifier randomly chooses a $k$-qubit stabilizer state and checks whether the quantum witness state is orthogonal to it.

Remark 4. If one takes $U=H$ in (5), the resulting projection operator projects onto the twodimensional subspace spanned by the vectors $|-\rangle\left|\gamma_{0}\right\rangle$ and $|+\rangle\left|\gamma_{1}\right\rangle$, where

$$
\left|\gamma_{0}\right\rangle=\cos (\pi / 8)|0\rangle+\sin (\pi / 8)|1\rangle \text { and }\left|\gamma_{1}\right\rangle=\sin (\pi / 8)|0\rangle-\cos (\pi / 8)|1\rangle
$$

are eigenvectors of $H$. This projection cannot be expressed as a sum of Clifford-Hamiltonian projections, which explains why we needed to replace $H$ with $H \otimes H$ in the proof above.

While considering this projection is not useful for proving Theorem 2, we do obtain from it a different result. In particular, we obtain an alternative proof of a result due to Morimae, Nagaj, and Schuch [39] establishing that single-qubit measurements and classical post-processing are sufficient for QMA verification. Reference [39] actually provides two proofs of this fact, one based on measurement-based quantum computation and the other based on a local-Hamiltonian problem type of approach similar to what we propose. While their local-Hamiltonian approach does not work for one-sided error (or $\mathrm{QMA}_{1}$ ) verifications, ours does (as does their measurement-based quantum computation proof).

\section{Description of the proof system}

In this section we describe our zero-knowledge proof system for the local Clifford-Hamiltonian problem. The main steps of the proof system are described in the subsections that follow, and the entire proof system is summarized in Figure 1. Properties of the proof system, including completeness, soundness, and the zero-knowledge property, are discussed in later sections of the paper.

As suggested previously, our proof system makes use of a bit commitment scheme, and in the interest of simplicity in explaining and analyzing the proof system we shall assume that this scheme is non-interactive. One could, however, replace this non-interactive commitment scheme by a different scheme (such as Naor's scheme with a 1-round commitment phase [40]). Throughout this section it is to be assumed that an instance of the $k$-local Clifford-Hamiltonian problem has been selected. The instance describes Clifford-Hamiltonian projections $H_{1}, \ldots, H_{m}$, each given by $H_{j}=C_{j}^{*}\left|0^{k}\right\rangle\left\langle 0^{k}\right| C_{j}$ for $k$-qubit Clifford operations $C_{1}, \ldots, C_{m}$, along with a specification of which of the $n$ qubits these projections act upon. The proof system does not refer to the parameters $p$ and $q$ in the description of the $k$-local Clifford Hamiltonian problem, as these parameters are only relevant to the performance of the proof system and not its implementation. It must be assumed, however, that the completeness parameter $2^{-p}$ is a negligible function of the entire problem instance size in order for the proof system to be zero-knowledge, and we will make this assumption hereafter.

\subsection{Prover's witness encoding}

Suppose $\mathrm{X}=\left(\mathrm{X}_{1}, \ldots, \mathrm{X}_{n}\right)$ is an $n$-tuple of single-qubit registers. These qubits are assumed to initially be in the prover's possession, and store an $n$-qubit quantum state $\rho$ representing a possible 


\section{Prover's encoding step:}

The prover selects a tuple $(t, \pi, a, b)$ uniformly at random, where $t=t_{1} \cdots t_{n}$ for $t_{1}, \ldots, t_{n} \in$ $\{0,+, \circlearrowright\}^{N}, \pi \in S_{2 N}$, and $a=a_{1} \cdots a_{n}$ and $b=b_{1} \cdots b_{n}$ for $a_{1}, \ldots, a_{n}, b_{1}, \ldots, b_{n} \in\{0,1\}^{2 N}$. The witness state contained in qubits $\left(\mathrm{X}_{1}, \ldots, \mathrm{X}_{n}\right)$ is encoded into qubit tuples

$$
\left(\mathrm{Y}_{1}^{1}, \ldots, \mathrm{Y}_{2 N}^{1}\right), \ldots,\left(\mathrm{Y}_{1}^{n}, \ldots, \mathrm{Y}_{2 N}^{n}\right)
$$

as described in the main text. These qubits are sent to the verifier, along with a commitment to the tuple $(\pi, a, b)$.

\section{Coin flipping protocol:}

The prover and verifier engage in a coin flipping protocol, choosing a string $r$ of a fixed length uniformly at random. This random string $r$ determines a Hamiltonian term $H_{r}=C_{r}^{*}\left|0^{k}\right\rangle\left\langle 0^{k}\right| C_{r}$ that is to be tested.

\section{Verifier's measurement:}

The verifier applies the Clifford operation $C_{r}$ transversally to the qubits

$$
\left(Y_{1}^{i_{1}}, \ldots, Y_{2 N}^{i_{1}}\right), \ldots,\left(Y_{1}^{i_{k}}, \ldots, Y_{2 N}^{i_{k}}\right),
$$

and measures all of these qubits in the standard basis, for $\left(i_{1}, \ldots, i_{k}\right)$ being the indices of the qubits upon which the Hamiltonian term $H_{r}$ acts nontrivially. The result of this measurement is sent to the prover.

\section{Prover's verification and response:}

The prover checks that the verifier's measurement results are consistent with the states of the trap qubits and the concatenated Steane code, aborting the proof system if not (causing the verifier to reject). In case the measurement results are consistent, the prover demonstrates that these measurement results are consistent with its prior commitment to $(\pi, a, b)$ and with the Hamiltonian term $H_{r}$, through a classical zero-knowledge proof system for the corresponding NP statement described in the main text. The verifier accepts or rejects accordingly.

Figure 1: Summary of the zero-knowledge proof system for the LCH problem 
witness for the instance of the $k$-LCH problem under consideration.

The first step of the proof system requires the prover to encode the state of $X$, using a scheme that consists of four steps. Throughout the description of these steps it is to be assumed that $N$ is a polynomially bounded function of the input size and is an even positive integer power of 7 . In effect, $N$ acts as a security parameter (for the zero-knowledge property of the proof system), and we take it to be an even power of 7 so that it may be viewed as a number of qubits that could arise from a concatenated Steane code allowing for a transversal application of Clifford operations, as described in Section A.6 (in the appendix). In particular, through an appropriate choice of $N$, one may guarantee that this code has any desired polynomial lower-bound for the minimum non-zero Hamming weight of its underlying classical code.

1. For each $i=1, \ldots, n$, the qubit $\mathrm{X}_{i}$ is encoded into qubits $\left(\mathrm{Y}_{1}^{i}, \ldots, \mathrm{Y}_{N}^{i}\right)$ by means of the concatenated Steane code. This results in the $N$-tuples

$$
\left(Y_{1}^{1}, \ldots, Y_{N}^{1}\right), \ldots,\left(Y_{1}^{n}, \ldots, Y_{N}^{n}\right)
$$

2. To each of the $N$-tuples in (11), the prover concatenates an additional $N$ trap qubits, with each trap qubit being initialized to one of the single qubit pure states $|0\rangle,|+\rangle$, or $|\circlearrowright\rangle$, selected independently and uniformly at random. This results in qubits

$$
\left(\mathrm{Y}_{1}^{1}, \ldots, \mathrm{Y}_{2 N}^{1}\right), \ldots,\left(\mathrm{Y}_{1}^{n}, \ldots, \mathrm{Y}_{2 N}^{n}\right) \text {. }
$$

The prover stores the string $t=t_{1} \cdots t_{n}$, for $t_{1}, \ldots, t_{n} \in\{0,+, \circlearrowright\}^{N}$ representing the randomly chosen states of the trap qubits.

3. A random permutation $\pi \in S_{2 N}$ is selected, and the qubits in each of the $2 N$-tuples (12) are permuted according to $\pi$. (Note that it is a single permutation $\pi$ that is selected and applied to all of the $2 \mathrm{~N}$-tuples simultaneously.)

4. The quantum one-time pad is applied independently to each qubit in (12) (after they are permuted in step 3$)$. That is, for $a_{i}, b_{i} \in\{0,1\}^{2 N}$ chosen independently and uniformly at random, the unitary transformation $X^{a_{i}} Z^{b_{i}}$ is applied to $\left(Y_{1}^{i}, \ldots, Y_{2 N}^{i}\right)$, and the strings $a_{i}$ and $b_{i}$ are stored by the prover, for each $i=1, \ldots, n$.

The randomness required by these encoding steps may be described by a tuple $(t, \pi, a, b)$, where $t$ is the string representing the states of the trap qubits described in step $2, \pi \in S_{2 N}$ is the permutation applied in step 3, and $a=a_{1} \cdots a_{n}$ and $b=b_{1} \cdots b_{n}$ are binary strings representing the Pauli operators applied in the one-time pad in step 4. After performing the above encoding steps, the prover sends the resulting qubits

$$
\mathrm{Y}=\left(\left(\mathrm{Y}_{1}^{1}, \ldots, \mathrm{Y}_{2 N}^{1}\right), \ldots,\left(\mathrm{Y}_{1}^{n}, \ldots, \mathrm{Y}_{2 N}^{n}\right)\right)
$$

along with a commitment

$$
z=\operatorname{commit}((\pi, a, b), s)
$$

to the tuple $(\pi, a, b)$, to the verifier. Here we assume that $s$ is a random string chosen by the prover that allows for this commitment. (It is not necessary for the prover to commit to the selection of the trap qubit states indicated by $t$, although it would not affect the properties of the proof system if it were modified so that the prover also committed to the trap qubit state selections.) 


\subsection{Verifier's random challenge}

Upon receiving the prover's encoded witness and commitment, the verifier issues a challenge: for a randomly selected index $j \in\{1, \ldots, m\}$, the verifier will check that the $j$-th Hamiltonian term

$$
H_{j}=C_{j}^{*}\left|0^{k}\right\rangle\left\langle 0^{k}\right| C_{j}
$$

is not violated. Generally speaking, the verifier's actions in issuing this challenge are as follows: for a certain collection of qubits, the verifier applies the Clifford operation $C_{j}$ transversally to those qubits, performs a measurement with respect to the standard basis, sends the outcomes to the prover, and then expects the prover to demonstrate that the obtained outcomes are valid (in the sense to be described later).

The randomly selected Hamiltonian term is to be determined by a binary string $r$, of a fixed length $\lceil\log m\rceil$, that should be viewed as being chosen uniformly at random. (In a moment we will discuss the random choice of $r$, which will be given by the output of a coin flipping protocol that happens to be uniform for honest participants.) It is not important exactly how the binary strings of length $\lceil\log m\rceil$ are mapped to the indices $\{1, \ldots, m\}$, so long as every index is represented by at least one string-so that for a uniformly chosen string $r$, each Hamiltonian term $j$ is selected with a nonnegligible probability. We will write $H_{r}$ and $C_{r}$ in place of $H_{j}$ and $C_{j}$, and refer to the Hamiltonian term determined by $r$, when it is convenient to do this.

It would be natural to allow the verifier to randomly determine which Hamiltonian term is to be tested-but, as suggested above, we will assume that the challenge is determined through a coin flipping protocol rather than leaving the choice to the verifier. More specifically, throughout the present subsection, it should be assumed that the random choice of the string $r$ that determines which challenge is issued is the result of independent iterations of a commitment-based coin-flipping protocol (i.e., the honest prover commits to a random $y_{i} \in\{0,1\}$, the honest verifier selects $z_{i} \in\{0,1\}$ at random, the prover reveals $y_{i}$, and the two participants agree that the $i$-th random bit of $r$ is $r_{i}=y_{i} \oplus z_{i}$ ). This guarantees (assuming the security of the commitment proto$\mathrm{col})$ that the choices are truly random, and greatly simplifies the analysis of the zero-knowledge property of the proof system. The use of such a protocol might not actually be necessary for the security of the proof system, but we leave the investigation of whether it is necessary to future work.

Now, let $\left(i_{1}, \ldots, i_{k}\right)$ denote the indices of the qubits upon which the Hamiltonian term determined by the random string $r$ acts nontrivially. The verifier applies the Clifford operation $C_{r}$ independently to each of the $k$-qubit tuples

$$
\left(Y_{1}^{i_{1}}, \ldots, Y_{1}^{i_{k}}\right), \ldots,\left(Y_{2 N}^{i_{1}}, \ldots, Y_{2 N}^{i_{k}}\right)
$$

which is equivalent to saying that $C_{r}$ is applied transversally to the tuples

$$
\left(\mathrm{Y}_{1}^{i_{1}}, \ldots, \mathrm{Y}_{2 N}^{i_{1}}\right), \ldots,\left(\mathrm{Y}_{1}^{i_{k}}, \ldots, \mathrm{Y}_{2 N}^{i_{k}}\right)
$$

that encode the qubits on which the Hamiltonian term $H_{r}$ acts nontrivially. The qubits (17) are then measured with respect to the standard basis, and the results are sent to the prover. We will let

$$
u_{i_{1}}, \ldots, u_{i_{k}} \in\{0,1\}^{2 N}
$$

denote the binary strings representing the verifier's standard basis measurement outcomes (or claimed outcomes) corresponding to the measurements of the tuples (17). 


\subsection{Prover's check and response}

Upon receiving the verifier's claimed measurement outcomes corresponding to the randomly selected Hamiltonian term, the prover first checks to see that these outcomes could indeed have come from the measurements specified above, and then tries to convince the verifier that these measurement outcomes are consistent with the selected term.

In more detail, suppose that the Hamiltonian term determined by $r$ has been challenged. As above, we assume that this term acts nontrivially on the $k$ qubits indexed by the $k$-tuple $\left(i_{1}, \ldots, i_{k}\right)$, and we will write

$$
u=u_{i_{1}} \cdots u_{i_{k}} \in\{0,1\}^{2 k N}
$$

to denote the verifier's claimed standard basis measurement outcomes.

To define the prover's check for this string, it will be helpful to first define a predicate $R_{r}$, which is a function of $t, \pi$, and $u$, and essentially represents the prover's check after it has made an adjustment to the verifier's response to account for the one-time pad. For each $i \in\left\{i_{1}, \ldots, i_{k}\right\}$, define strings $y_{i}, z_{i} \in\{0,1\}^{N}$ so that

$$
\pi\left(y_{i} z_{i}\right)=u_{i} .
$$

The predicate $R_{r}$ takes the value 1 if and only if these two conditions are met:

1. $y_{i} \in \mathcal{D}_{N}$ for every $i \in\left\{i_{1}, \ldots, i_{k}\right\}$, and $y_{i} \in \mathcal{D}_{N}^{1}$ for at least one index $i \in\left\{i_{1}, \ldots, i_{k}\right\}$.

2. $\left\langle z_{i_{1}} \cdots z_{i_{k}}\left|C_{r}^{\otimes N}\right| t_{i_{1}} \cdots t_{i_{k}}\right\rangle \neq 0$.

(Here we have written $\left|t_{i_{1}} \cdots t_{i_{k}}\right\rangle$ to denote the pure state of $k N$ qubits obtained by tensoring the states $|0\rangle,|+\rangle$, and $|\circlearrowright\rangle$ in this most natural way.) The first condition concerns measurement outcomes corresponding to non-trap qubits, and reflects the condition that these measurement outcomes are proper encodings of binary values-but not all of which encode 0 . The second condition concerns the consistency of the verifier's measurements with the trap qubits.

Next, we will define a predicate $Q_{r}$, which is a function of the variables $t, \pi, a, b$, and $u$, where $t, \pi$, and $u$ are as above and $a, b \in\{0,1\}^{2 n N}$ refer to the strings used for the one-time pad. The predicate $Q_{r}$ represents the prover's actual check, in the case that the Hamiltonian term determined by $r$ has been selected, including an adjustment to account for the one-time pad. Let $c_{1}, \ldots, c_{n}, d_{1}, \ldots, d_{n} \in\{0,1\}^{2 N}$ be the unique strings for which the equation

$$
C_{r}^{\otimes 2 N}\left(X^{a_{1}} Z^{b_{1}} \otimes \cdots \otimes X^{a_{n}} Z^{b_{n}}\right)=\alpha\left(X^{c_{1}} Z^{d_{1}} \otimes \cdots \otimes X^{c_{n}} Z^{d_{n}}\right) C_{r}^{\otimes 2 N}
$$

holds for some choice of $\alpha \in\{1, i,-1,-i\}$. The Clifford operation $C_{r}$ acts trivially on those qubits indexed by strings outside of the set $\left\{i_{1}, \ldots, i_{k}\right\}$, so it must be the case that $c_{i}=a_{i}$ and $d_{i}=b_{i}$ for $i \notin\left\{i_{1}, \ldots, i_{k}\right\}$, but for those indices $i \in\left\{i_{1}, \ldots, i_{k}\right\}$ it may be the case that $c_{i} \neq a_{i}$ and $d_{i} \neq b_{i}$. We will also write $c=c_{1} \cdots c_{n}$ and $d=d_{1} \cdots d_{n}$ for the sake of convenience. Given a description of the Clifford operation $C_{r}$ it is possible to efficiently compute $c$ and $d$ from $a$ and $b$. Having defined $c$ and $d$, we may now express the predicate $Q_{r}$ as follows:

$$
Q_{r}(t, \pi, u, a, b)=R_{r}\left(t, \pi, u \oplus c_{i_{1}} \cdots c_{i_{k}}\right) .
$$

In essence, the predicate $Q_{r}$ checks the validity of the verifier's claimed measurement results by first adjusting for the one-time pad, then referring to $R_{r}$.

The prover evaluates the predicate $Q_{r}$, and aborts the proof system if the predicate evaluates to 0 (as this is indicative of a dishonest verifier). Otherwise, the prover aims to convince the verifier that the measurement outcomes $u$ are consistent with the prover's encoding, and also that they are 
not in violation of the Hamiltonian term $H_{r}$. It does this specifically by engaging in a classical zeroknowledge proof system for the following NP statement: there exists a random string $s$ and an encoding key $(t, \pi, a, b)$ such that (i) commit $((\pi, a, b), s)$ matches the prover's initial commitment $z$, and (ii) $Q_{r}(t, \pi, u, a, b)=1$.

It will be convenient later, in the analysis of the proof system, to sometimes view $r$ as being an input to the predicates defined above. Specifically, we define predicates

$$
Q(r, t, \pi, a, b, u)=Q_{r}(t, \pi, a, b, u) \quad \text { and } \quad R(r, t, \pi, u)=R_{r}(t, \pi, u)
$$

for this purpose.

\section{Completeness and soundness of the proof system}

It is evident that the proof system described in the previous section is complete. For a given instance of the local Clifford Hamiltonian problem, if the prover and verifier both behave honestly, as suggested in the description of the proof system, the verifier will accept with precisely the same probability that would be obtained by randomly selecting a Hamiltonian term, measuring the original $n$-qubit witness state against the corresponding projection, and accepting or rejecting accordingly. For a positive problem instance, this acceptance probability is at least $1-2^{-p}$ (for every choice of a random string $r$ ).

Next we will consider the soundness of the proof system. We will prove that on a negative instance of the problem, the honest verifier must reject with nonnegligible probability. The prover initially sends to the verifier the qubits

$$
\left(\mathrm{Y}_{1}^{1}, \ldots, \mathrm{Y}_{2 N}^{1}\right), \ldots,\left(\mathrm{Y}_{1}^{n}, \ldots, \mathrm{Y}_{2 N}^{n}\right)
$$

along with a commitment $z=\operatorname{commit}((\pi, a, b), s)$ to a tuple $(\pi, a, b)$. We have assumed that the commitment is perfectly binding, so there is a well-defined tuple $(\pi, a, b)$ that is determined by the prover's commitment $z$. We may assume without loss of generality that this tuple has the proper form (meaning that $\pi \in S_{2 N}$ is a permutation and $a$ and $b$ are binary strings of length $2 n N$, as specified in the description of the proof system), as a commitment to a string not of this form must lead to rejection with high probability in all cases. Let $\xi$ be the state of the qubits

$$
\left(\mathrm{Y}_{1}^{1}, \ldots, \mathrm{Y}_{N}^{1}\right), \ldots,\left(\mathrm{Y}_{1}^{n}, \ldots, \mathrm{Y}_{N}^{n}\right)
$$

that is obtained by inverting the quantum one-time pad with respect to the strings $a$ and $b$, inverting the permutation of each of the tuples (24) with respect to the permutation $\pi$, and discarding the last $N$ qubits within each tuple (i.e., the trap qubits). For an honest prover, the state $\xi$ would be the state obtained by encoding the original witness state using the concatenated Steane codealthough in general it cannot be assumed that $\xi$ arises in this way. Although the verifier is not capable of recovering the state $\xi$ on its own, because it does not know $(\pi, a, b)$, it will nevertheless be helpful to refer to the state $\xi$ for the purposes of establishing the soundness condition of the proof system.

We will define a collection of $N$-qubit projections operators and a channel from $N$ qubits to one that will be useful for establishing soundness. First, let

$$
\Pi_{0}=\sum_{x \in \mathcal{D}_{N}^{0}}|x\rangle\langle x| \quad \text { and } \quad \Pi_{1}=\sum_{x \in \mathcal{D}_{N}^{1}}|x\rangle\langle x|,
$$


where $\mathcal{D}_{N}^{0}$ and $\mathcal{D}_{N}^{1}$ are subsets of $\{0,1\}^{N}$ representing classical code words of the concatenated Steane code. A standard basis measurement of any qubit encoded using this code will necessarily yield an outcome in one of these two sets: an encoded $|0\rangle$ state yields an outcome in $\mathcal{D}_{N}^{0}$, and an encoded $|1\rangle$ state yields an outcome in $\mathcal{D}_{N}^{1}$. The projections $\Pi_{0}$ and $\Pi_{1}$ therefore correspond to these two possibilities, while the projection operator $\mathbb{1}-\left(\Pi_{0}+\Pi_{1}\right)$ corresponds to the situation in which a standard basis measurement has yielded a result outside of the classical code space $\mathcal{D}_{N}=\mathcal{D}_{N}^{0} \cup \mathcal{D}_{N}^{1}$. Also define projections

$$
\Delta_{0}=\frac{\mathbb{1}^{\otimes N}+Z^{\otimes N}}{2} \quad \text { and } \quad \Delta_{1}=\frac{\mathbb{1}^{\otimes N}-Z^{\otimes N}}{2},
$$

which are the projections onto the spaces spanned by all even- and odd-parity standard basis states, respectively. It holds that $\Pi_{0} \leq \Delta_{0}$ and $\Pi_{1} \leq \Delta_{1}$, as the codewords in $\mathcal{D}_{N}^{0}$ all have even parity and the codewords in $\mathcal{D}_{N}^{1}$ all have odd parity. Finally, define a channel $\Xi_{N}$, mapping $N$ qubits to 1 qubit, as follows:

$$
\Xi_{N}(\sigma)=\frac{\left\langle\mathbb{1}^{\otimes N}, \sigma\right\rangle \mathbb{1}+\left\langle X^{\otimes N}, \sigma\right\rangle X+\left\langle Y^{\otimes N}, \sigma\right\rangle Y+\left\langle Z^{\otimes N}, \sigma\right\rangle Z}{2},
$$

for every $N$-qubit operator $\sigma$. It is evident that this mapping preserves trace, and is completely positive when $N \equiv 1(\bmod 4)$, which holds because $N$ is an even power of 7 . One may observe that the adjoint mapping to $\Xi_{N}$ is given by

$$
\Xi_{N}^{*}(\tau)=\frac{\langle\mathbb{1}, \tau\rangle \mathbb{1}^{\otimes N}+\langle X, \tau\rangle X^{\otimes N}+\langle Y, \tau\rangle Y^{\otimes N}+\langle Z, \tau\rangle Z^{\otimes N}}{2}
$$

and satisfies

$$
\Xi_{N}^{*}(|0\rangle\langle 0|)=\Delta_{0} \quad \text { and } \quad \Xi_{N}^{*}(|1\rangle\langle 1|)=\Delta_{1} .
$$

Now, consider the state $\rho=\Xi_{N}^{\otimes n}(\xi)$ of the qubits $\left(\mathrm{X}_{1}, \ldots, \mathrm{X}_{n}\right)$ that is obtained from $\xi$ when $\Xi_{N}$ is applied independently to each of the $N$-tuples of qubits in (25). We will prove that the verifier must reject with nonnegligible probability for a given choice of $r$ provided that $\rho$ violates the corresponding Hamiltonian term $H_{r}$. Because every $n$-qubit state creates a nonnegligible violation in at least one Hamiltonian term for a negative problem instance, this will suffice to prove the soundness of the proof system.

For each random string $r$ generated by the coin flipping procedure, one may define a measurement on the state $\xi$ that corresponds to the verifier's actions and final decision to accept or reject given this choice of $r$, assuming the prover behaves optimally after the coin flipping and the verifier's measurement take place. Specifically, corresponding to the Hamiltonian term $H_{r}=C_{r}^{*}\left|0^{k}\right\rangle\left\langle 0^{k}\right| C_{r}$, acceptance is represented by a projection operator $\Lambda_{r}$ on the qubits

$$
\left(\mathrm{Y}_{1}^{i_{1}}, \ldots, \mathrm{Y}_{N}^{i_{1}}\right), \ldots,\left(\mathrm{Y}_{1}^{i_{k}}, \ldots, \mathrm{Y}_{N}^{i_{k}}\right)
$$

defined as follows:

$$
\Lambda_{r}=\sum_{\substack{z \in\{0,1\}^{k} \\ z \neq 0^{k}}}\left(C_{r}^{\otimes N}\right)^{*}\left(\Pi_{z_{1}} \otimes \cdots \otimes \Pi_{z_{k}}\right)\left(C_{r}^{\otimes N}\right) .
$$

The probability the verifier rejects, for a given choice of $r$, is therefore at least $1-\left\langle\Lambda_{r}, \xi\right\rangle$. Because $\Pi_{0} \leq \Delta_{0}$ and $\Pi_{1} \leq \Delta_{1}$, the probability of rejection is therefore at least

$$
1-\sum_{\substack{z \in\{0,1\}^{k} \\ z \neq 0^{k}}}\left\langle\left(C_{r}^{\otimes N}\right)^{*}\left(\Delta_{z_{1}} \otimes \cdots \otimes \Delta_{z_{k}}\right)\left(C_{r}^{\otimes N}\right), \xi\right\rangle=\left\langle\left(C_{r}^{\otimes N}\right)^{*}\left(\Delta_{0} \otimes \cdots \otimes \Delta_{0}\right)\left(C_{r}^{\otimes N}\right), \xi\right\rangle .
$$


By considering properties of the channel $\Xi_{N}$, we conclude that the verifier rejects with probability at least

$$
\begin{aligned}
& \left\langle\left(C_{r}^{\otimes N}\right)^{*}\left(\Xi_{N}^{*}(|0\rangle\langle 0|) \otimes \cdots \otimes \Xi_{N}^{*}(|0\rangle\langle 0|)\right)\left(C_{r}^{\otimes N}\right), \xi\right\rangle \\
= & \left\langle\left(\Xi_{N}^{\otimes k}\right)^{*}\left(C_{r}^{*}\left|0^{k}\right\rangle\left\langle 0^{k}\right| C_{r}\right), \xi\right\rangle=\left\langle C_{r}^{*} \mid 0^{k}\right\rangle\left\langle 0^{k} \mid C_{r}, \Xi_{N}^{\otimes k}(\xi)\right\rangle=\left\langle H_{r}, \rho\right\rangle .
\end{aligned}
$$

Here we have used the observation that

$$
\Xi_{N}^{\otimes k}\left(C^{\otimes N} \sigma\left(C^{\otimes N}\right)^{*}\right)=C \Xi_{N}^{\otimes k}(\sigma) C^{*}
$$

for every $k$-qubit Clifford operation $C$ and every $k N$-qubit state $\sigma$, which may be verified directly by considering the definition of $\Xi_{N}$.

Intuitively speaking, the argument above shows that whatever state a malicious prover sends in the first message, one can essentially decode that state with respect to a highly simplified variant of the encoding scheme (after peeling off the quantum one-time pad and discarding the trap qubits), recovering a state that would pass the Hamiltonian energy test with at least the same probability as the verifier's acceptance probability in our zero-knowledge proof system. Because this probability must be bounded away from 1 on average for any no-instance of the problem, we obtain a soundness guarantee for the proof system.

\section{Zero-knowledge property of the proof system}

In this section we will prove that the proof system described in Section 3 is quantum computational zero-knowledge, assuming that the commitment scheme used in the proof system is unconditionally binding and quantum computationally concealing. The proof has several steps, to be presented below, but first we will summarize the main technical goal of the proof.

Figure 2 shows a diagram of the interaction between the honest participants in the proof system. A cheating verifier aiming to extract knowledge from the prover might, of course, not follow the prescribed actions of the honest verifier. In particular, the cheating verifier may take a quantum register as input, store quantum information in between its actions, and output a quantum register. Figure 3 illustrates such a cheating verifier interacting with the honest prover. The goal of the proof is to demonstrate that, for any cheating verifier of the form suggested by Figure 3, there exists an efficient simulator that implements a channel from $Z_{0}$ to $Z_{3}$ that is computationally indistinguishable from the channel implemented by the cheating verifier and prover interaction. In particular, the simulator does not have access to the witness state $\rho$.

\section{Step 1: simulating the coin flipping protocol}

By the results of [12], there must exist an efficient simulator $S_{1}$ for the interaction of $V_{1}^{\prime}$ with $P_{1}$. To be more precise, for $S_{1}$ being given an input of the same form as $V_{1}^{\prime}$, along with a uniformly chosen random string $r$ of the length required by our proof system, the resulting action is quantum computationally indistinguishable from $V_{1}^{\prime}$ interacting with $P_{1}$. Figure 4 illustrates the process that is obtained by performing this substitution. As the simulator $S_{1}$ together with the true random string generator is computationally indistinguishable from the interaction between $V_{1}^{\prime}$ and $P_{1}$, the process illustrated in Figure 4 is computationally indistinguishable from the process illustrated in Figure 3. It therefore suffices for us to prove that the process illustrated in Figure 4 can be efficiently simulated (without access to the witness state $\rho$ ). 


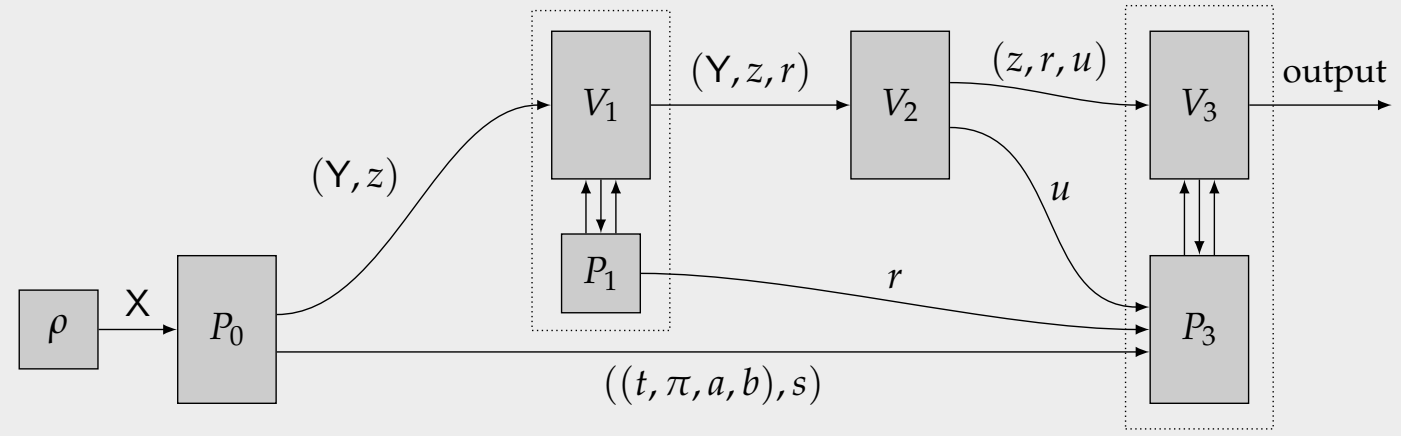

Figure 2: The interaction between honest participants. The prover's quantum witness $\rho$ is encoded into $Y$ together with the encoding key $(t, \pi, a, b)$ by the prover's action $P_{0}$. The string $z$ represents the prover's commitment to $(\pi, a, b)$ and the string $s$ represents random bits used by the prover to implement this commitment. The string $r$ represents the random bits generated by the coin flipping protocol, which is depicted within the dotted rectangle on the left. The string $u$ represents the verifier's standard basis measurements for a subset of the qubits of $Y$ determined by the challenge corresponding to the random string $r$. The classical zero-knowledge protocol is depicted within the dotted rectangle on the right.

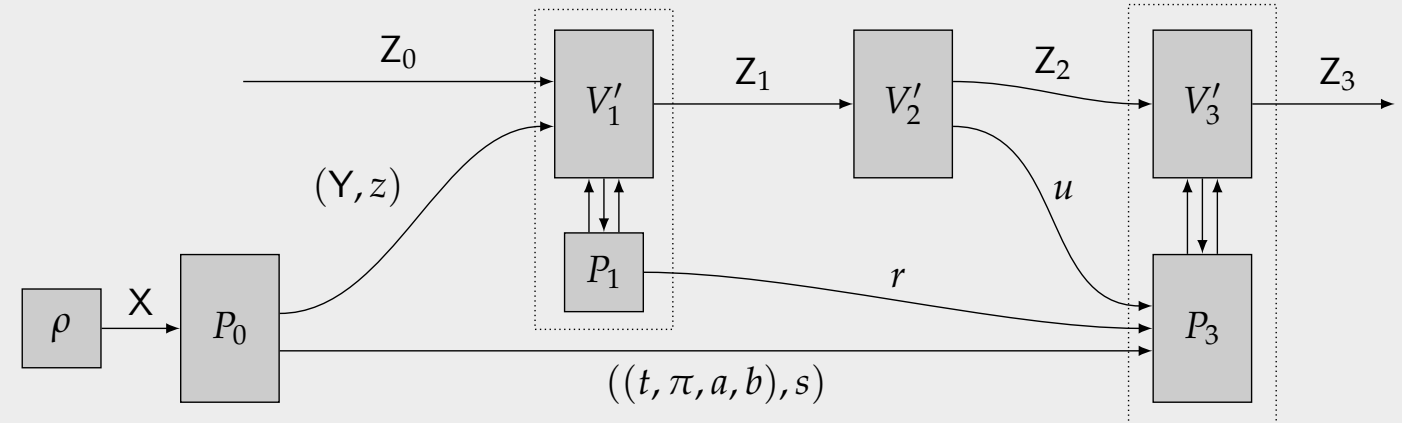

Figure 3: A potentially dishonest verifier takes an auxiliary quantum register $Z_{0}$ as input, may store quantum information (represented by registers $Z_{1}$ and $Z_{2}$ ), and outputs quantum information stored in register $Z_{3}$.

\section{Step 2: simulating the classical zero-knowledge protocol}

In the next step of the proof, we replace the interaction between a cheating verifier $V_{3}^{\prime}$ and the prover $P_{3}$ in the classical zero-knowledge protocol by an efficient simulation.

The prover holds an encoding key $(t, \pi, a, b)$ along with a random string $s$ it has used to commit to the tuple $(\pi, a, b)$. The commitment $z=\operatorname{commit}((\pi, a, b), s)$ was sent to the verifier, together with the encoding register $Y$, in the first step of the proof system. The verifier sends a string $u$ that, in the honest case, represents the output of a measurement of some subset of the qubits of $Y$ with respect to the standard basis, after the transversal application of a Clifford operation 


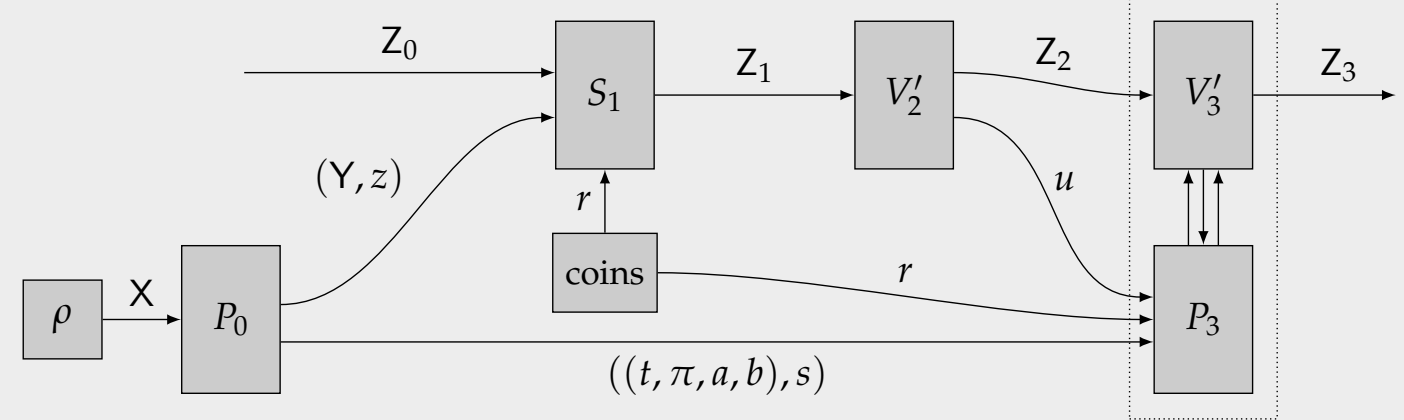

Figure 4: The interaction corresponding to the execution of the coin flipping protocol has been replaced by a simulator $S_{1}$ along with a true random string generator (labeled coins).

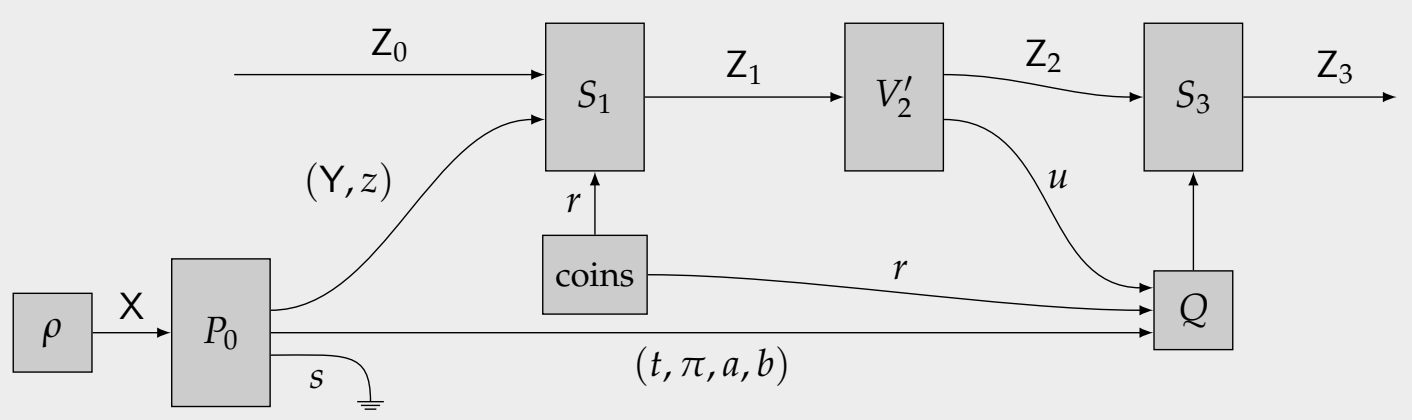

Figure 5: The interaction corresponding to the execution of the classical zero-knowledge protocol has been replaced by a simulator $S_{3}$ along with the predicate $Q$. It is assumed that when the output of $Q$ is 0 , the simulator $S_{3}$ behaves as the cheating verifier $V_{3}^{\prime}$ would when the prover aborts the proof system. The string $s$ produced by $P_{0}$ in forming the commitment to $(\pi, a, b)$ is discarded.

depending on the random choice of $r$. The statement that the honest prover aims to prove in the classical zero-knowledge protocol is that there exists an encoding key $(t, \pi, a, b)$ along with a string $s$ such that $z=\operatorname{commit}((\pi, a, b), s)$ and $Q(r, t, \pi, a, b, u)=1$. The honest prover always holds an encoding key $(t, \pi, a, b)$ and a binary string $s$ for which $z=\operatorname{commit}((\pi, a, b), s)$, and if it is the case that $Q(r, t, \pi, a, b, u)=0$, the honest prover aborts. By the assumption that the classical zero-knowledge protocol is indeed computational zero-knowledge, there must therefore exist an efficient simulator $S_{3}$ so that the process described in Figure 5 is computationally indistinguishable from the one described by Figure 4 . Note that the string $s$ used by $P_{0}$ to form the commitment $z=\operatorname{commit}((\pi, a, b), s)$ can be discarded immediately after $P_{0}$ is run. 


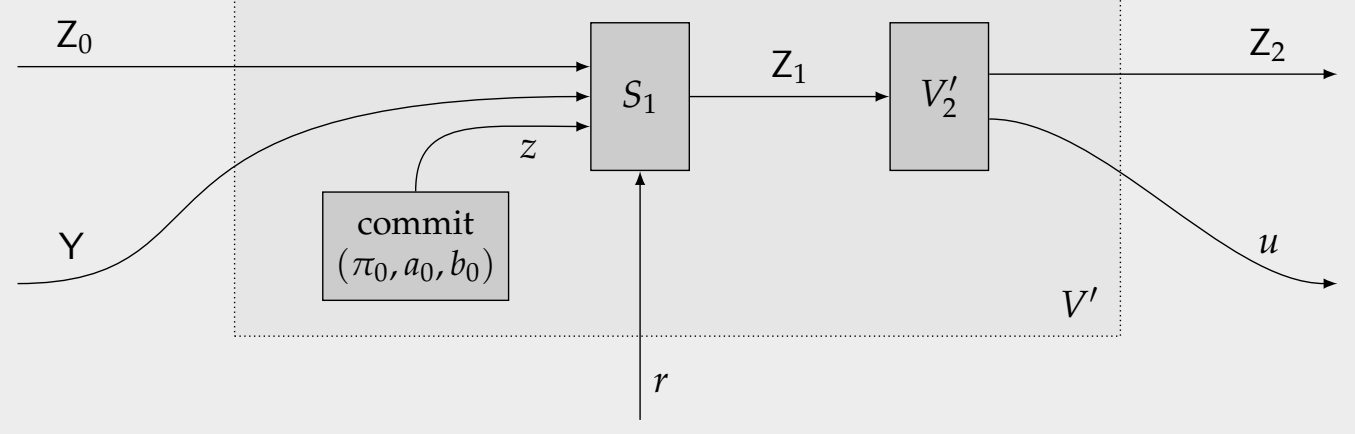

Figure 6: The commitment to a fixed tuple $\left(\pi_{0}, a_{0}, b_{0}\right)$, the simulator $S_{1}$, and the dishonest verifier action $V_{2}^{\prime}$ may be merged into a single efficiently implementable action $V^{\prime}$ that represents an attack against the encoding scheme.

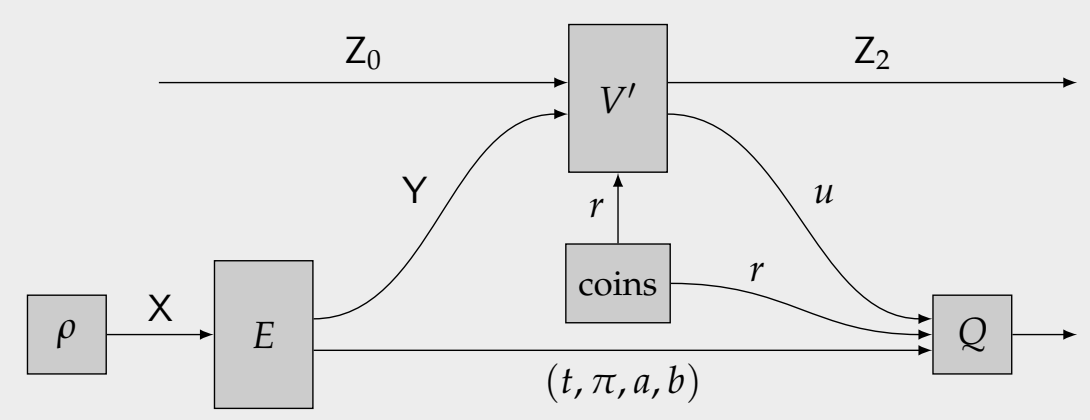

Figure 7: A cheating verifier $V^{\prime}$ aims to extract knowledge from the encoding of a register $X$.

\section{Step 3: eliminating the commitment}

The next step is to eliminate the commitment. Because it is assumed that the commitment scheme is quantum computationally concealing, and the commitment is never revealed by the process described in Figure 5, this process is computationally indistinguishable from a similar process in which the commitment $z$ is made to a fixed choice of a tuple $\left(\pi_{0}, a_{0}, b_{0}\right)$, independent of the prover's encoding key. In particular, one may take $\pi_{0}$ to be the identity permutation and $a_{0}$ and $b_{0}$ to be all-zero strings of length $2 n N$. One may now consider the commitment to this fixed tuple $\left(\pi_{0}, a_{0}, b_{0}\right)$, together with the simulator $S_{1}$ and the cheating verifier action $V_{2}^{\prime}$, to form a single, efficiently implementable action $V^{\prime}$ as suggested by Figure 6.

The interaction between this new action $V^{\prime}$ and the prover's encoding, the random string generator, and the predicate $Q$, as is illustrated in Figure 7, may now be considered. If it is proved that the channel implemented by this process can be efficiently simulated, then it will follow that the channel implemented by the process described in Figure 5 can be efficiently simulated (in a computationally indistinguishable sense). This is so because the composition of the process illustrated in Figure 7 with the efficiently implementable simulator $S_{3}$ is computationally indistinguishable from the process described in Figure 5. 


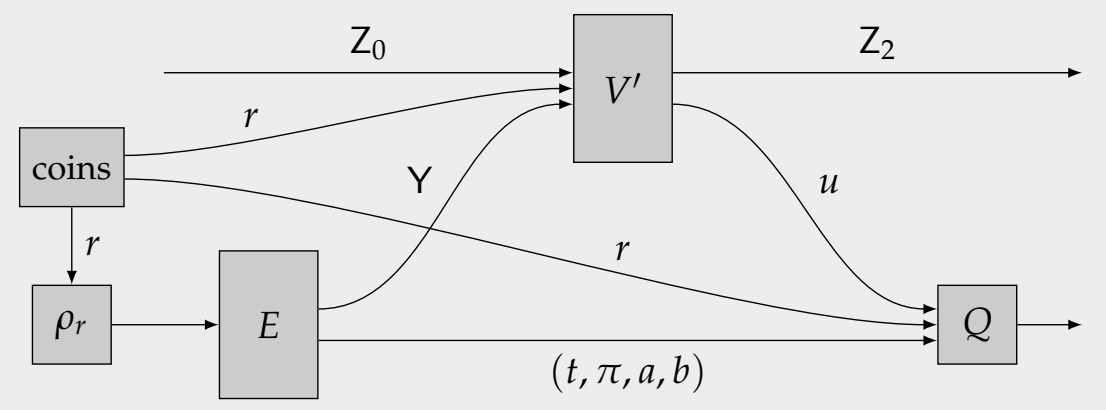

Figure 8: The simulation of the process shown in Figure 7 is nearly identical to that process, except that it uses the random string $r$ to encode a state $\rho_{r}$ that is guaranteed to pass the challenge corresponding to $r$, rather than encoding the witness state $\rho$.

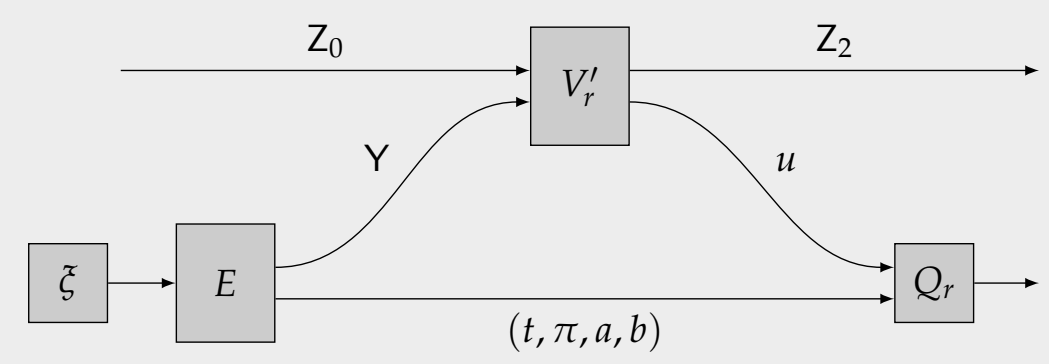

Figure 9: An arbitrary $n$-qubit state $\xi$ is encoded, and the cheating verifier $V^{\prime}$ and predicate $Q$ for a fixed choice of a string $r$ interact as depicted. It will be proved that the channels obtained by substituting $\rho$ and $\rho_{r}$ for $\xi$ are approximately equal.

\section{Step 4: simulating an attack on the encoding scheme}

It therefore suffices for us to prove that, for any efficiently implementable action $V^{\prime}$, the channel implemented by the process described by Figure 7 can be efficiently simulated. In fact, it will be possible to efficiently simulate this channel with statistical accuracy, not just in a computationally indistinguishable sense. This is not surprising: we have claimed that the computational zeroknowledge property of our proof system is based on a computationally concealing commitment scheme, and the uses of the commitment scheme have all been eliminated from consideration by the steps above.

At this point we may describe the simulator directly: it is illustrated in Figure 8, and it represents the most straightforward approach to obtaining a simulator. This simulator differs from the process described in Figure 7 in that it uses the output of the random string generator to choose a quantum state that, once encoded, passes the randomly selected challenge with certainty. It is trivial to efficiently prepare such a state given the string $r$. It remains to prove that the channel implemented by the simulator described in Figure 8 is indistinguishable from the channel implemented by the process described in Figure 7. By convexity it suffices to prove that this is so for every fixed choice of the string $r$. 


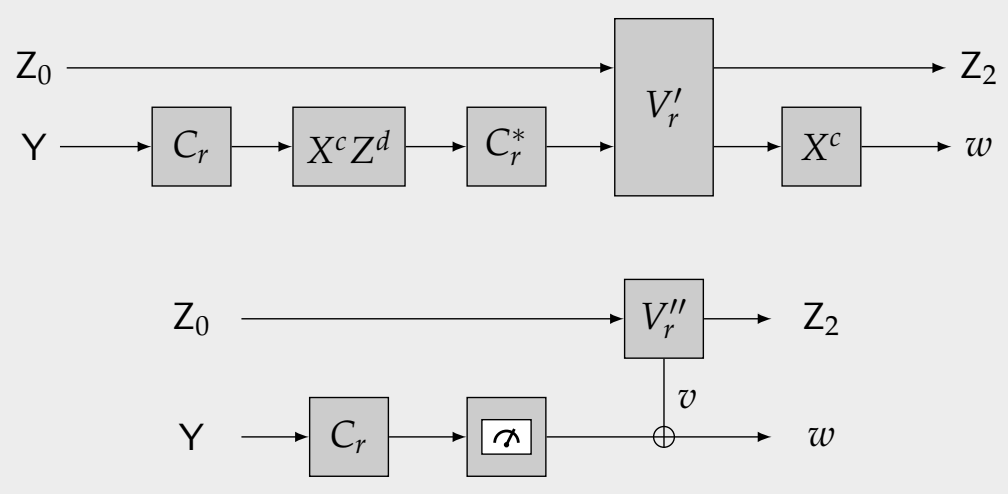

Figure 10: The prover's one-time pad merged with the cheating verifier operation $V_{r}^{\prime}$. Averaging over random choices of $c$ and $d$ results in a process that can alternatively be described as illustrated in the lower diagram. In this process, $V_{r}^{\prime \prime}$ represents a so-called quantum instrument, which transforms $Z_{0}$ into $Z_{2}$ and produces a classical measurement outcome. In this case, this classical measurement outcome is XORed onto the string produced by a standard basis measurement. (In this figure and the next, one should interpret $C_{r}$ and $C_{r}^{*}$ as referring to the transversal application of the corresponding Clifford operation.)

With this goal in mind, consider the process described in Figure 9, in which an arbitrary state $\xi$ is encoded (corresponding either to $\rho$ or $\rho_{r}$ in Figures 7 and 8), and the string $r$ is fixed (which has been indicated by the substitution of $V_{r}^{\prime}$ and $Q_{r}$ for $V^{\prime}$ and $Q$, respectively). We will prove that the channel implemented by any such process can have only a limited dependence on the state $\xi$.

More specifically, let us assume that $\xi_{0}$ and $\xi_{1}$ are arbitrary $n$-qubit states, let $p_{0}$ and $p_{1}$ denote the probabilities with which these two states would pass the challenge determined by $r$ (for an honest prover and verifier pair), and let $\Psi_{0}$ and $\Psi_{1}$ denote the channels from $Z_{0}$ to $Z_{2}$ together with the output bit of the predicate $Q_{r}$ that are implemented by the process shown in Figure 9 when $\xi_{0}$ or $\xi_{1}$ is substituted for $\xi_{\text {, respectively. }}$

We claim that if the difference $\left|p_{0}-p_{1}\right|$ is negligible, then the distance $\left\|\Psi_{0}-\Psi_{1}\right\|_{\diamond}$ is also negligible. The two steps that follow establish that this claim is true. By the assumption that the prover initially holds a witness state $\rho$ that satisfies every Hamiltonian term with probability exponentially close to 1 , this will complete the proof.

\section{Step 5: twirling the cheating verifier}

To prove the fact suggested above regarding the channel implemented by Figure 9, we will naturally need to make use of the specific properties of the encoding scheme, which has not played an important role in the analysis thus far. The first step is to recognize that the effect of the prover's one time pad is to $t_{\text {wirl }}{ }^{3}$ the verifier as Figure 10 illustrates.

In greater detail, the last step of the encoding process is the quantum one-time pad: the prover independently chooses one of the Pauli operations $\mathbb{1}, X, Z$, or $X Z$ for each qubit of $Y$ and applies

\footnotetext{
${ }^{3}$ The term twirl is commonly used in quantum information theory to describe a process whereby a symmetrization over a collection of randomly chosen unitary operations has a particular effect on a state or channel. Twirled states and channels often take on a significantly simpler form than the original state or channel prior to twirling.
} 


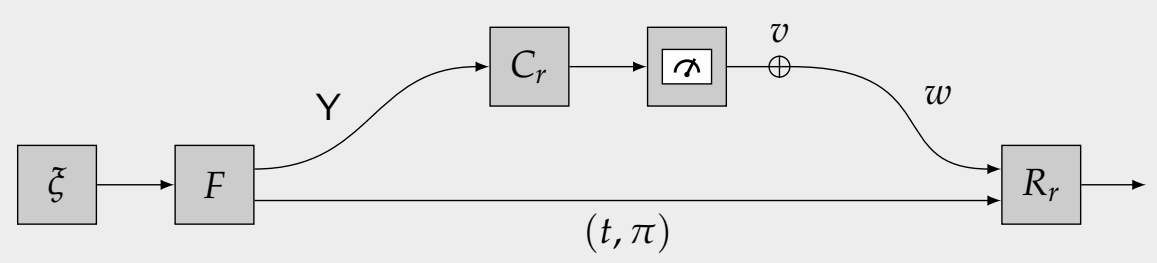

Figure 11: An XOR attack against the prover's encoding scheme without the one-time pad. The transformation $F$ denotes the first three steps of the prover's encoding scheme.

that operation, storing the randomly selected strings $a, b \in \Sigma^{2 N n}$. With respect to the Clifford operation $C_{r}$ associated with the randomly selected challenge (determined by the string $r$ ), the prover computes the pair $(c, d)$ for which it holds that

$$
X^{a} Z^{b}=\left(C_{r}^{\otimes 2 N}\right)^{*} X^{c} Z^{d}\left(C_{r}^{\otimes 2 N}\right) .
$$

The first step when computing the predicate $Q_{r}$ is the application of $X^{c}$ to the string $u$, which is supposed to represent the outcome of a standard basis measurement of a subset of the qubits after the transversal application of $C_{r}$ to the corresponding qubits in the register $Y$. The resulting string $w=u \oplus c$ is then fed into the predicate $R_{r}$ described previously. Merging the Clifford operation $C_{r}^{*}$ with the cheating verifier operation $V_{r}^{\prime}$, then averaging over $c$ and $d$ chosen uniformly at random (which is equivalent to averaging over $a$ and $b$ chosen uniformly at random), one obtains a process of the form illustrated in the lower diagram in Figure 10.

By the observation we have just made, it suffices to consider processes of the form described in Figure 11, in which an $n$-qubit state $\xi$ is encoded as described by the first three steps in the prover's encoding procedure (but not including the one-time pad), the Clifford operation $C_{r}$ (for a fixed choice of $r$ ) is applied transversally to the resulting register, and the qubits on which those transversal Clifford operations act are measured with respect to the standard basis. For some arbitrary but fixed string $v$, the XOR of the outcome of this measurement with $v$ is fed into the predicate $R_{r}$. The process outputs a single bit, obtained by evaluating the predicate $R_{r}$.

\section{Step 6: encoding security under XOR attacks}

Now let us return to the claim made previously, in which $\xi_{0}$ and $\xi_{1}$ represent $n$-qubit states, $p_{0}$ and $p_{1}$ denote the probabilities with which these two states would pass the challenge determined by $r$ (for an honest prover and verifier pair), and $\Psi_{0}$ and $\Psi_{1}$ denote the channels implemented by the process shown in Figure 9 when $\xi_{0}$ or $\xi_{1}$ is substituted for $\xi$, respectively. If it is the case that the distribution of output bits obtained by substituting $\xi_{0}$ and $\xi_{1}$ for $\xi$ in Figure 11 have negligible statistical difference, then it follows that the difference $\left\|\Psi_{0}-\Psi_{1}\right\|_{\diamond}$ is also negligible. It therefore remains to argue that the distributions obtained by substituting $\xi_{0}$ and $\xi_{1}$ into Figure 11 have negligible statistical difference.

Before finishing off the last step of the analysis, it is helpful to consider the possible outcomes of the measurement, the definition of $R_{r}$, and the behavior of the procedure described in Figure 11 when $v=0 \cdots 0$ is the all-zero string. For any choice of $\xi$, the measurement is guaranteed to yield a string of length $2 k N$ taking the form $u_{i_{1}} \cdots u_{i_{k}}$, where $u_{i_{1}}, \ldots, u_{i_{k}} \in\{0,1\}^{2 N}$ and $\left(i_{1}, \ldots, i_{k}\right)$ index the qubits on which $C_{r}$ acts nontrivially. With respect to a particular choice of $(t, \pi)$, if we define 
strings $y_{i}, z_{i} \in\{0,1\}^{N}$, for each $i \in\left\{i_{1}, \ldots, i_{k}\right\}$, so that

$$
\pi\left(y_{i} z_{i}\right)=u_{i}
$$

then these two conditions will necessarily be met:

1. $y_{i} \in \mathcal{D}_{N}$ for every $i \in\left\{i_{1}, \ldots, i_{k}\right\}$, and

2. $\left\langle z_{i_{1}} \cdots z_{i_{k}}\left|C_{r}^{\otimes N}\right| t_{i_{1}} \cdots t_{i_{k}}\right\rangle \neq 0$.

Moreover, in the case that $r$ determines a Hamiltonian term challenge, the event that $y_{i} \in \mathcal{D}_{N}^{1}$ for at least one index $i \in\left\{i_{1}, \ldots, i_{k}\right\}$ is equivalent to $\xi$ passing this challenge. Thus, in the case that $v=0 \cdots 0$, the process described in Figure 11 outputs the bit 1 with precisely the probability that an honest prover and verifier pair would result in acceptance, assuming the prover's initial state is $\xi$ and $r$ is selected as a random string determining the challenge.

Now let us assume that $v$ is a nonzero string, and let us consider two cases: the first is that the Hamming weight $|v|_{1}$ of $v$ satisfies $|v|_{1}<K$, for $K$ being the minimum Hamming weight of a nonzero codeword in $\mathcal{D}_{N}$, and the second case is that $|v|_{1} \geq K$.

If it is the case that $|v|_{1}<K$, then there are two possible ways that the value of the predicate $R_{r}$ could change, in comparison to the case $v=0 \cdots 0$. In both cases, if there is a change, it must be from 1 to 0 , caused by one of the two conditions above becoming violated. The first case is that one or more bits in one of the codewords $y_{i_{1}}, \ldots, y_{i_{k}}$ is flipped, causing the first condition listed above to become violated. The second case is that a measurement outcome for the trap qubits is obtained that potentially violates the second condition. Note that it is not possible that the first condition remains satisfied, but the Hamiltonian term challenge condition that $y_{i} \in \mathcal{D}_{N}^{1}$ for at least one index $i \in\left\{i_{1}, \ldots, i_{k}\right\}$ changes, as such a change would require at least $K$ bit-flips to cause a logical change in valid codewords. It is unimportant for the purposes of the analysis to determine the probability with which one of the two conditions becomes violated, except to observe that it is independent of $\xi$. (In somewhat more detail, the string $v$ may be written as $v=v_{i_{1}} \cdots v_{i_{k}}$, and the probability that neither of the two conditions is affected is given by the probability that $\pi^{-1}\left(v_{i}\right)$ places no $1 \mathrm{~s}$ within the first $N$ bits or over a trap qubit left in a standard basis state within the second $N$ bits, for a random choice of $\pi$ and for each $i \in\left\{i_{1}, \ldots, i_{k}\right\}$.)

If it is the case that $|v|_{1} \geq K$, then there is a possibility that, in comparison to the functioning of the process for $v=0 \cdots 0$, the Hamiltonian term challenge condition that $y_{i} \in \mathcal{D}_{N}^{1}$ for at least one index $i \in\left\{i_{1}, \ldots, i_{k}\right\}$ could be affected. That is, $v$ has enough Hamming weight to affect the logical values represented by the codewords $y_{i_{1}}, \ldots, y_{i_{k}}$. However, as we will show, the assumption that $|v|_{1} \geq K$ necessarily leads to a negligible probability that the second condition remains satisfiedfor a string $v$ having Hamming weight $K$ or higher, the probability that none of the traps is sprung is exponentially small. In order to argue that this is so, we require the following simple lemma.

Lemma 3. Let $k$ be a positive integer, let $C$ be a Clifford operation on $k$ qubits, and let $j \in\{1, \ldots, k\}$. There exists a string $t \in\{0,+, \circlearrowright\}^{k}$, a bit $a \in\{0,1\}$, and pure states $\left|\phi_{0}\right\rangle$ and $\left|\phi_{1}\right\rangle$ on $j-1$ qubits and $k-j$ qubits, respectively, so that

$$
C|t\rangle=\left|\phi_{0}\right\rangle|a\rangle\left|\phi_{1}\right\rangle \text {. }
$$

Equivalently, there is a choice of $t$ so that the j-th qubit of $C|t\rangle$ is left in a standard basis state.

Proof. The lemma is equivalent to the existence of a string $t$ so that $|t\rangle$ is an eigenvector of the operator

$$
C^{*}\left(\mathbb{1}^{\otimes(j-1)} \otimes Z \otimes \mathbb{1}^{\otimes(k-j)}\right) C
$$


As the Clifford group normalizes the Pauli group, the operator (39) is a scalar multiple of a tensor product of Pauli operators and identity operators. The lemma follows from the observation that $t$ may be chosen so that each $\left|t_{1}\right\rangle, \ldots,\left|t_{k}\right\rangle$ is an eigenvector of the Pauli operator in the corresponding position.

By this lemma, one finds that for a random choice of $t \in\{0,+, \circlearrowright\}^{k N}$, and for any $k$-qubit Clifford operation $C$ applied transversally to $|t\rangle$, each qubit is left in a standard basis state with probability at least $3^{-k}$, and for any choice of $N$ or fewer qubits acted on by distinct Clifford operations these events are independent. In greater detail, if the qubits

$$
\left(Z_{1}^{1}, \ldots, Z_{1}^{k}\right), \ldots,\left(Z_{N}^{1}, \ldots, Z_{N}^{k}\right)
$$

are initialized to the state $|t\rangle$, for $t \in\{0,+, \circlearrowright\}^{k N}$ chosen uniformly at random, and the $k$-qubit Clifford operation $C$ is applied independently to each $k$-tuple of qubits, then each qubit is left in a standard basis state with probability at least $3^{-k}$, and the states of the $k$-tuples of qubits are independent.

Now we return to the analysis for a string $v$ of length $2 k N$ having Hamming weight at least $K$. By virtue of the fact just mentioned, it is straightforward to obtain a negligible upper-bound on the probability for the process described in Figure 11 to output 1 . As this event requires that a random choice of the permutation $\pi$ leaves none of the 1-bits of $v$ in positions corresponding to trap qubits left in standard basis states by the transversal action of $C_{r}$, we find that the probability to output 1 is exponentially small in $K$. In particular, this probability is at most

$$
\left(1-\frac{1}{3^{k+1}}\right)^{K / k}=\exp (-\varepsilon(k) K)
$$

where $\varepsilon(k)$ denotes a positive real number depending on $k$ but not $K$.

From a consideration of the two cases just presented, we may conclude the following. Suppose as before that $\xi_{0}$ and $\xi_{1}$ are $n$-qubit states that may be substituted for $\xi$ in Figure 11, and that the probabilities $p_{0}$ and $p_{1}$ for these states to pass the challenge determined by a fixed choice of $r$ have negligible difference. Let us write $q_{0}(v)$ and $q_{1}(v)$, respectively, to denote the probability that the process described in Figure 11 outputs 1 . As noted before, it holds that $p_{0}=q_{0}(0 \cdots 0)$ and $p_{1}=q_{1}(0 \cdots 0)$. For any choice of $v$ satisfying $|v|_{1}<K$, we have that $q_{0}(v)=\beta(v) q_{0}(0 \cdots 0)$ and $q_{1}(v)=\beta(v) q_{1}(0 \cdots 0)$ for $\beta(v) \in(0,1)$ that is independent of $\xi_{0}$ and $\xi_{1}$. Finally, for any choice of $v$ satisfying $|v|_{1} \geq K$, we have that $q_{0}(v)$ and $q_{1}(v)$ are both negligible. It therefore follows that the difference $\left|q_{0}(v)-q_{1}(v)\right|$ is negligible in all cases, which completes the proof.

\section{Conclusion}

This paper gives a zero-knowledge proof system for any problem in QMA assuming the existence of a quantum computationally concealing and unconditionally binding commitment scheme. Such a commitment scheme can be obtained assuming quantum-secure one-way permutations [1] (or injections more generally) or a quantum-secure pseudo-random generator [40] that could potentially be based on one-way functions that are hard to invert for any quantum polynomial time algorithm $[29,43,52]$. We conclude with a few open questions and directions for future work.

1. Our proof system inherits the soundness error of the most straightforward verification procedure for the local Clifford-Hamiltonian problem, which is to randomly select a Hamiltonian 
term and perform a measurement corresponding to it. When an arbitrary QMA problem is reduced to the local Hamiltonian problem, the resulting soundness error may potentially be large (polynomially bounded away from 1). Can one obtain a zero-knowledge proof system for any QMA problem with small soundness error while maintaining the other features of our proof system (e.g., constant round of communications)?

We note that if a prover has polynomially many copies of a valid quantum witness, then a parallel repetition of our proof system may yield a constant round zero-knowledge proof system having small soundness error for any QMA problem-but this would require a parallel repetition result concerning zero-knowledge proof systems for NP secure against quantum attacks. Analogous results for zero-knowledge proofs for NP against classical attacks are known $[15,19]$, but they involve sophisticated rewinding arguments for which known quantum rewinding techniques do not seem to be applicable.

2. Are there natural formalizations of proofs of quantum knowledge? Roughly speaking, one would expect such a notion to require that whenever a prover is able to prove the validity of a statement, one could construct a knowledge extractor that can extract a quantum witness given access to such a prover. It seems plausible that our proof system could be adapted to such a notion, although we have not investigated this notion in depth.

3. We have considered an encoding scheme for quantum states that ensures the secrecy of the state and allows for the transversal application of constant-size Clifford operations and measurement in the computational basis. It is an interesting open question to extend our encoding scheme, or to design a new one, so that it can support transversally applying a larger family of quantum operations.

4. Finally, we make one further remark on an abstract view of our proof system. Classically speaking, one can imagine a "commit-and-open" primitive where a sender commits to a message $m$, and later opens sufficient information so that a receiver can test a property $\mathcal{P}(\cdot)$ on $m$, and nothing more. For example, $\mathcal{P}$ can be an NP-relation $R(x, \cdot)$ that checks if message $m$ is a valid witness. This can be implemented easily by a standard commitment scheme and during the opening phase, the sender and receiver run a zero-knowledge proof of $R(x, m)=1$ instead of the standard opening. Our proof system, which combines a commitment scheme and classical zero-knowledge proofs for NP, can be viewed as a quantum analogue. Namely, we commit to a witness state and open just enough information to verify that some reduced density of the witness state falls into a specific subspace. We can only deal with properties of a very special form, and it is an interesting direction for future work to generalize and find applications of this sort of primitive.

\section{Acknowledgments}

We thank Michael Beverland, Sevag Gharibian, David Gosset, Yi-Kai Liu and Bei Zeng for helpful conversations. A. B. and J. W. are supported in part by Canada's NSERC. F. S. is supported in part by Cryptoworks21, Canada's NSERC and CIFAR. 


\section{A Preliminaries}

This section summarizes some of the notation, definitions, and known facts concerning quantum information and computation, cryptography, and other topics that are used throughout the paper. We refer to $[34,41,48]$ for further details on the theory of quantum information and computation. Further information on classical zero-knowledge and cryptography can be found in $[17,18]$.

\section{A.1 Basic terminology}

Throughout the paper we let $\Sigma=\{0,1\}$ denote the binary alphabet, and only consider strings, promise problems, and complexity classes over this alphabet. For a string $x \in \Sigma^{*},|x|$ denotes its length. A function $g: \mathbb{N} \rightarrow \mathbb{N}$ is a polynomially bounded function if there exists a deterministic polynomial-time Turing machine $M_{g}$ that outputs $1^{g(n)}$ on input $1^{n}$ for every non-negative integer $n$. A function $f: \mathbb{N} \rightarrow[0, \infty)$ is said to be negligible if, for every polynomially bounded function $g$, it holds that $f(n)<1 / g(n)$ for all but finitely many values of $n$.

\section{A.2 Quantum information basics}

When we refer to a quantum register in this paper, we simply mean a collection of qubits that we wish to view as a single unit and to which we give some name. Names of registers will always be uppercase letters in a sans serif font, such as $X, Y$, and $Z$. The finite dimensional complex Hilbert spaces associated with registers will be denoted by capital script letters such as $\mathcal{X}, \mathcal{Y}$, and $\mathcal{Z}$, using the same letter in the two different fonts to denote a quantum register and its corresponding space for convenience. Dirac notation is used to express vectors in Hilbert spaces and linear mappings between them in a standard way.

For a given space $\mathcal{X}$, we let $\mathrm{L}(\mathcal{X})$ denote the set of all linear mappings (or operators) from $\mathcal{X}$ to itself. The identity element of $\mathrm{L}(\mathcal{X})$ is denoted $\mathbb{1}_{\mathcal{X}}$, or just as $\mathbb{1}$ when $\mathcal{X}$ can be taken as implicit. The inner product between operators $A$ and $B$ is defined as $\langle A, B\rangle=\operatorname{Tr}\left(A^{*} B\right)$.

Quantum states are represented by density operators, which are positive semidefinite operators having unit trace. A linear map $\Phi: \mathrm{L}(\mathcal{X}) \rightarrow \mathrm{L}(\mathcal{Y})$ is said to be a channel if it is both completely positive and trace-preserving. Channels are mappings from density operators to density operators that, in principle, represent physically realizable operations. A measurement is described by a collections of positive semidefinite operators $\left\{M_{j}\right\}$ such that $\sum_{j} M_{j}=\mathbb{1}$, with the probability that the measurement on state $\rho$ results in outcome $j$ being given by $\left\langle M_{j}, \rho\right\rangle$

We review a few definitions of norms on operators, which are used to discuss the distinguishability of quantum states and channels. The trace norm of an operator $X \in \mathrm{L}(\mathcal{X})$ is defined as $\|X\|_{1}=\operatorname{Tr} \sqrt{X^{*} X}$. For any linear map $\Phi: \mathrm{L}(\mathcal{X}) \rightarrow \mathrm{L}(\mathcal{Y})$, the diamond norm (or completely bounded trace norm) $[3,33,34]$ is defined as

$$
\|\Phi\|_{\diamond}=\max \left\{\left\|\left(\Phi \otimes \mathbb{1}_{\mathrm{L}(\mathcal{W})}\right)(X)\right\|_{1}: X \in \mathrm{L}(\mathcal{X} \otimes \mathcal{W}),\|X\|_{1} \leq 1\right\}
$$

where $\mathcal{W}$ is any space with dimension equal to that of $\mathcal{X}$. (The value remains the same for any choice of $\mathcal{W}$, provided its dimension is at least that of $\mathcal{X}$.)

\section{Quantum gates and circuits}

A quantum circuit is an acyclic network of quantum gates connected by wires. The quantum gates represent quantum channels while the wires represent qubits on which the channels act. 
We will refer to two types of quantum circuits in this paper: unitary quantum circuits and general quantum circuits. By unitary quantum circuits we mean circuits composed of unitary gates (such as the ones described below) chosen from some finite gate set. General quantum circuits are composed of gates that may correspond to channels that are not necessarily unitary. It is sufficient for the purposes of this paper that we consider just two simple non-unitary gates: ancillary gates, which input nothing and output a qubit in the $|0\rangle$ state; and erasure gates, which input one qubit and output nothing (and correspond to the channel described by the trace mapping). As is described elsewhere [3,50], arbitrary channels mapping one register to another can always be approximated arbitrarily closely by quantum circuits whose gates include a universal collection of unitary gates together with ancillary and erasure gates. The size of a quantum circuit is the number of gates in the circuit plus the number of qubits on which it acts.

We will refer to the following well-known single-qubit unitary gates:

1. Pauli gates:

$$
X:|a\rangle \mapsto|1-a\rangle \quad \text { and } \quad Z:|a\rangle \mapsto(-1)^{a}|a\rangle,
$$

for each $a \in\{0,1\}$, as well as $Y=i X Z$.

2. Hadamard gate:

$$
H:|a\rangle \mapsto \frac{1}{\sqrt{2}}|0\rangle+\frac{(-1)^{a}}{\sqrt{2}}|1\rangle,
$$

for each $a \in\{0,1\}$.

3. Phase gate:

$$
P:|a\rangle \mapsto i^{a}|a\rangle
$$

for each $a \in\{0,1\}$.

In addition, for any $k$-qubit unitary quantum gate $U$ we define the controlled- $U$ gate as

$$
\Lambda(U):|a\rangle|x\rangle \mapsto|a\rangle U^{a}|x\rangle,
$$

for each $a \in\{0,1\}$ and $x \in\{0,1\}^{k}$.

The $k$-qubit Pauli group is the group containing all unitary operators of the form

$$
\alpha U_{1} \otimes \cdots \otimes U_{k}
$$

where $\alpha \in\{1, i,-1,-i\}$ and $U_{1}, \ldots, U_{k} \in\{\mathbb{1}, X, Y, Z\}$, where $\mathbb{1}$ denotes the single-qubit identity operation. Elements of this group are also referred to as Pauli operations. If $a, b \in\{0,1\}^{k}$ are binary strings of length $k$, then we write

$$
X^{a}=X^{a_{1}} \otimes \cdots \otimes X^{a_{k}} \quad \text { and } \quad Z^{b}=Z^{b_{1}} \otimes \cdots \otimes Z^{b_{k}}
$$

to denote the Pauli operations obtained from these strings as indicated.

Channels that can be expressed as convex combinations of unitary channels that correspond to Pauli operations are called Pauli channels. An example of Pauli channels that is relevant to this paper is the completely depolarizing channel

$$
\Omega(\rho)=\frac{1}{4} \sum_{a, b \in\{0,1\}}\left(X^{a} Z^{b}\right) \rho\left(X^{a} Z^{b}\right)^{*}=\frac{1}{2},
$$

for any single-qubit density operator $\rho$. We thus see that the effect of $\Omega$ is to completely randomize the state of a single-qubit system. By treating a random choice of a pair $(a, b)$ as a secret key, we 
obtain a quantum generalization of the one-time pad, known as the quantum one-time pad [4]. When the channel is performed independently on $k$ qubits, the effect is given by

$$
\Omega^{\otimes k}(\rho)=2^{-k} \mathbb{1} \otimes \cdots \otimes \mathbb{1}
$$

for every $k$-qubit density operator $\rho$. The quantum one-time pad generalizes naturally to any choice of the number $k$.

Sometimes it will be convenient to consider quantum circuits that implement measurements. When we refer to a measurement circuit, we mean any general quantum circuit, followed by a measurement of all of its output qubits with respect to the standard basis. If $Q$ is a measurement circuit that is applied to a collection of qubits in the state $\rho$, then $Q(\rho)$ is interpreted as a string-valued random variable describing the resulting measurement. We will only need to refer to measurement circuits outputting a single bit in this paper.

A $k$-qubit Clifford circuit is any unitary quantum circuit on $k$ qubits whose gates are drawn from the set $\{H, P, \Lambda(X)\}$ containing Hadamard, phase, and controlled-not gates. (It is common that one also allows Pauli gates to be included in this set for convenience. Given that $X=H P P H$ and $Z=P P$, there is no generality lost in using the smaller gate set in the definition.) The set of all unitary operators that can be described by $k$-qubit Clifford circuits forms a finite group known as the Clifford group. Up to scalar multiples, the $k$-qubit Clifford group is the normalizer of the $k$-qubit Pauli group: if $U$ is a $k$-qubit unitary operator for which it holds that $U V U^{*}$ is an element of the $k$-qubit Pauli group for every $k$-qubit Pauli group element $V$, then $U=\alpha C$ for $\alpha \in \mathbb{C}$ satisfying $|\alpha|=1$ and $C$ being a $k$-qubit Clifford group element. Given the description of a $k$-qubit Pauli group element $V$ and a $k$-qubit Clifford circuit $C$, one can efficiently compute a description of the $k$-qubit Pauli group element $C V C^{*}[26]$.

Clifford circuits are not universal for quantum computation. Two examples (among other known examples) of universal gate sets are the following:

1. Hadamard, phase, and Toffoli gates: $\{H, P, \Lambda(\Lambda(X))\}$.

2. Hadamard and controlled-phase gates: $\{H, \Lambda(P)\}$.

The first of these choices is sometimes easier to work with, but we will make use of the fact that the second gate set is universal in the paper.

\section{A.3 Polynomial-time generated families of quantum circuits and QMA}

Any quantum circuit with gates drawn from a fixed, finite gate set can be encoded as a binary string, with respect to a variety of possible encoding schemes. The specific details of such encoding schemes are not important within the context of this paper, so we will leave it to the reader to imagine that a sensible and efficient encoding scheme for quantum circuits has been selected, relative to whatever gate set is under consideration. It should be assumed, of course, that a circuit's size and its encoding length are polynomially related.

For any infinite set of binary strings $S \subseteq\{0,1\}^{*}$, a collection $\left\{V_{x}: x \in S\right\}$ of quantum circuits is said to be polynomial-time generated if there exists a deterministic polynomial-time Turing machine that, on input $x \in S$, outputs an encoding of $V_{x}$. The assumptions on encoding schemes suggested above imply that, if $\left\{V_{x}: x \in S\right\}$ is a polynomial-time generated collection, then $V_{x}$ must have size polynomial in $|x|$.

Next we will define the complexity class QMA, which is commonly viewed as the most natural quantum generalization of NP. 
Definition 4. A promise problem $A=\left(A_{\text {yes }}, A_{\text {no }}\right)$ is contained in the complexity class $\mathrm{QMA}_{\alpha, \beta}$ if there exists a polynomial-time generated collection

$$
\left\{V_{x}: x \in A_{\text {yes }} \cup A_{\text {no }}\right\}
$$

of quantum circuits and a polynomially bounded function $p$ possessing the following properties:

1. For every string $x \in A_{\text {yes }} \cup A_{\text {no, }}$ one has that $V_{x}$ is a measurement circuit taking $p(|x|)$ input qubits and outputting a single bit.

2. Completeness. For all $x \in A_{\text {yes, }}$, there exists a $p(|x|)$-qubit state $\rho$ such that $\operatorname{Pr}\left(V_{x}(\rho)=1\right) \geq \alpha$.

3. Soundness. For all $x \in A_{\text {no, }}$ and every $p(|x|)$-qubit state $\rho$, it holds that $\operatorname{Pr}\left(V_{x}(\rho)=1\right) \leq \beta$.

In this definition, $\alpha, \beta \in[0,1]$ may be constant values or functions of the length of the input string $x$. When they are omitted, it is to be assumed that they are $\alpha=2 / 3$ and $\beta=1 / 3$. Known error reduction methods [34,37] imply that a wide range of selections of $\alpha$ and $\beta$ give rise to the same complexity class. In particular, QMA coincides with $\mathrm{QMA}_{\alpha, \beta}$ for $\alpha=1-2^{-q(|x|)}$ and $\beta=2^{-q(|x|)}$, for any polynomially bounded function $q$.

\section{A.4 Quantum computational indistinguishability and zero-knowledge}

Next we review notions of quantum state and channel discrimination, as well as zero-knowledge in a quantum setting (as defined in [49]).

We first specify what it means for two collections of quantum states to be quantum computationally indistinguishable. The definition that follows may be viewed as being a non-uniform notion of quantum computational indistinguishability, as it places no uniformity conditions on quantum circuits and allows for an auxiliary quantum state $\sigma$ to assist in the task of state discrimination.

Definition 5 (Quantum computationally indistinguishable states). Suppose that $S \subseteq\{0,1\}^{*}$ is an infinite set of binary strings, $r$ is a polynomially bounded function, and $\rho_{x}$ and $\xi_{x}$ are states on $r(|x|)$ qubits for each $x \in S$. The collections $\left\{\rho_{x}: x \in S\right\}$ and $\left\{\xi_{x}: x \in S\right\}$ are quantum computationally indistinguishable if, for every choice of polynomially bounded functions $s$ and $k$, any measurement circuit $Q$ of size $s(|x|)$, and any choice of a $k(|x|)$-qubit state $\sigma$, it holds that

$$
\left|\operatorname{Pr}\left[Q\left(\rho_{x} \otimes \sigma\right)=1\right]-\operatorname{Pr}\left[Q\left(\xi_{x} \otimes \sigma\right)=1\right]\right| \leq \varepsilon(|x|)
$$

for all $x \in S$, for a negligible function $\varepsilon$.

The notion extends naturally to distinguishing collections of channels, as the following definition makes precise.

Definition 6 (Quantum computationally indistinguishable channels). Suppose that $S \subseteq\{0,1\}^{*}$ is an infinite set of binary strings, $q$ and $r$ are polynomially bounded functions, and $\Phi_{x}$ and $\Psi_{x}$ are channels from $q(|x|)$ qubits to $r(|x|)$ qubits for each $x \in S$. The collections $\left\{\Phi_{x}: x \in S\right\}$ and $\left\{\Psi_{x}: x \in S\right\}$ are quantum computationally indistinguishable if, for every choice of polynomially bounded functions $s$ and $k$, every state $\sigma$ on $q(|x|)+k(|x|)$ qubits, and every measurement circuit $Q$ on $r(|x|)+k(|x|)$ qubits having size $s(|x|)$, one has that

$$
\left|\operatorname{Pr}\left[Q\left(\left(\Phi_{x} \otimes \mathbb{1}\right)(\sigma)\right)=1\right]-\operatorname{Pr}\left[Q\left(\left(\Psi_{x} \otimes \mathbb{1}\right)(\sigma)\right)=1\right]\right| \leq \varepsilon(|x|)
$$

for every $x \in S$, for a negligible function $\varepsilon$. 
We will also make use of statistical notions of indistinguishability for states and channels, which are defined as follows.

Definition 7 (Statistically indistinguishable states). Suppose that $S \subseteq\{0,1\}^{*}$ is an infinite set of binary strings, $r$ is a polynomially bounded function, and $\rho_{x}$ and $\xi_{x}$ are states on $r(|x|)$ qubits for each $x \in S$. The collections $\left\{\rho_{x}: x \in S\right\}$ and $\left\{\xi_{x}: x \in S\right\}$ are statistically indistinguishable if

$$
\frac{1}{2}\left\|\rho_{x}-\xi_{x}\right\|_{1} \leq \varepsilon(|x|)
$$

for all $x \in S$, for a negligible function $\varepsilon$.

Definition 8 (Statistically indistinguishable channels). Suppose that $S \subseteq\{0,1\}^{*}$ is an infinite set of binary strings, $q$ and $r$ are polynomially bounded functions, and $\Phi_{x}$ and $\Psi_{x}$ are channels from $q(|x|)$ qubits to $r(|x|)$ qubits for each $x \in S$. The collections $\left\{\Phi_{x}: x \in S\right\}$ and $\left\{\Psi_{x}: x \in S\right\}$ are statistically indistinguishable if

$$
\frac{1}{2}\left\|\Phi_{x}-\Psi_{x}\right\|_{\diamond} \leq \varepsilon(|x|)
$$

for all $x \in S$, for a negligible function $\varepsilon$.

Next we review the definition of quantum computational zero-knowledge proof systems as defined in [49]. Let $(P, V)$ be a quantum or classical interactive proof system for a promise problem $A$. An arbitrary (possibly malicious) verifier $V^{\prime}$ is any quantum computational process that interacts with $P$ according to the structural specification of $(P, V)$. Similar to the classical notion of auxiliary input zero-knowledge, a verifier $V^{\prime}$ will take, in addition to the input string $x$, an auxiliary input, and produce some output. This is crucial for the composition of zero-knowledge proof systems. The most general situation allowed by quantum information theory is that both the auxiliary input and the output are quantum, meaning that the verifier operates on quantum registers whose initial state is arbitrary and may be entangled with some external system. Also similar to the classical case, we will assume that for any given polynomial-time verifier $V^{\prime}$ there exist polynomially bounded functions $q$ and $r$ that determine the number of auxiliary input qubits and output qubits of $V^{\prime}$. To say that $V^{\prime}$ is a polynomial-time verifier means that the entire action of $V^{\prime}$ must be described by some polynomial-time generated family of quantum circuits.

The interaction of a verifier $V^{\prime}$ with $P$ on input $x$ induces some channel from the verifier's $q(|x|)$ auxiliary input qubits to $r(|x|)$ output qubits. Let $\mathcal{W}$ denote the vector space corresponding to the auxiliary input qubits, let $\mathcal{Z}$ denote the space corresponding to the output qubits, and let $\Phi_{x}: \mathrm{L}(\mathcal{W}) \rightarrow \mathrm{L}(\mathcal{Z})$ denote the resulting channel induced by the interaction of $V^{\prime}$ with $P$ on input $x$. A simulator $S$ for a given verifier $V^{\prime}$ is described by a polynomial-time generated family of general quantum circuits that agrees with $V^{\prime}$ on the functions $q$ and $r$ representing the number of auxiliary input qubits and output qubits respectively. Such a simulator does not interact with $P$, but simply induces a channel that we will denote by $\Psi_{x}: \mathrm{L}(\mathcal{W}) \rightarrow \mathrm{L}(\mathcal{Z})$ on each input $x$.

Definition 9 (Quantum computational zero-knowledge). An interactive proof system $(P, V)$ for a promise problem $A$ is quantum computational zero-knowledge if, for every polynomial-time generated quantum verifier $V^{\prime}$, there exists a polynomial-time generated quantum simulator $S$ that satisfies the following requirements.

1. The verifier $V^{\prime}$ and simulator $S$ agree on the polynomially bounded functions $q$ and $r$ that specify the number of auxiliary input qubits and output qubits, respectively.

2. Let $\Phi_{x}$ be the channel that results from the interaction between $V^{\prime}$ and $P$ on input $x$, and let $\Psi_{x}$ be the channel induced by the simulator $S$ on input $x$, both as described above. Then the collections $\left\{\Phi_{x}: x \in A_{\text {yes }}\right\}$ and $\left\{\Psi_{x}: x \in A_{\text {yes }}\right\}$ are quantum computationally indistinguishable. 


\section{A.5 Cryptographic Tools}

Here we introduce a few cryptographic building blocks that are useful in our proof system. We emphasize that, as is typical in the classical setting, we formulate all computational security properties (e.g., concealing in a commitment scheme) with respect to non-uniform quantum adversaries. This is inherited from the definition of quantum computational indistinguishability. This gives more stringent security requirements and is also crucial in security proofs.

\section{Commitment schemes}

For the sake of simplicity, we describe a commitment scheme that is non-interactive, i.e., all messages are going from a sender to a receiver. A similar definition can be derived for interactive schemes.

Definition 10 (Quantum computationally secure commitment schemes). A quantum computationally secure commitment scheme for an alphabet $\Gamma$ is a collection of polynomial-time computable functions $\left\{f_{n}: n \in \mathbb{N}\right\}$ taking the form

$$
f_{n}: \Gamma \times\{0,1\}^{p(n)} \rightarrow\{0,1\}^{q(n)},
$$

for polynomially bounded functions $p$ and $q$, such that the following conditions hold:

1. Unconditionally binding property. For every choice of $n \in \mathbb{N}, a, b \in \Gamma$, and $r, s \in\{0,1\}^{p(n)}$, one has that $f_{n}(a, r)=f_{n}(b, s)$ implies $a=b$.

2. Quantum computationally concealing property. For every $a \in \Gamma$ and $n \in \mathbb{N}$, define

$$
\rho_{a, n}=\frac{1}{2^{p(n)}} \sum_{r \in\{0,1\}^{p(n)}}\left|f_{n}(a, r)\right\rangle\left\langle f_{n}(a, r)\right| .
$$

For every choice of $a, b \in \Gamma$ the ensembles $\left\{\rho_{a, n}: n \in \mathbb{N}\right\}$ and $\left\{\rho_{b, n}: n \in \mathbb{N}\right\}$ are quantum computationally indistinguishable.

To commit to a string, one can independently use the commitment described above bit by bit. Such a commitment scheme can be constructed based on certain quantum intractability assumptions. As shown in [1], it suffices to have quantum-resistant one-way permutations, which are permutations that can be computed efficiently on a classical computer but are hard to invert for both classical and quantum polynomial-time algorithms. The same commitment scheme remains quantum-secure based on a slightly weaker assumption of quantum-resistant injective one-way functions. Naor showed a commitment scheme with a two-message commit phase [40] which will be quantum-secure [28], assuming one uses a pseudo-random generator whose output is quantum computationally indistinguishable from a truly random string ${ }^{4}$.

Based on such a quantum-secure commitment scheme, we can obtain the other two essential cryptographic building blocks in our protocol: a zero-knowledge proof system for NP and a coinflipping protocol, both secure against quantum adversaries.

\footnotetext{
${ }^{4}$ It has been stated informally (see e.g., [43,52]) that the pseudo-random generator by Håstad et al. [29] based on oneway functions would remain quantum-secure, so long as the one-way functions are resistant to any polynomial-time quantum inverting algorithms.
} 


\section{Zero-knowledge proof for NP}

Watrous showed that [49] the GMW 3-Coloring protocol [21] remains zero-knowledge in the presence of quantum verifiers, assuming a statistically binding and quantum computationally hiding commitment scheme. This means that we have a classical zero-knowledge proof protocol for any NP language that is secure against any polynomial-time quantum verifiers.

\section{Coin-flipping}

A coin-flipping protocol is an interactive process that allows two parties to jointly toss random coins. It is not necessary for us to consider this notion generally, as we only make use of one specific coin-flipping protocol, namely Blum's coin-flipping protocol [8] in which an honest prover commits to a random $y \in\{0,1\}$, the honest verifier selects $z \in\{0,1\}$ at random, the prover reveals $y$, and the two participants agree that the random bit generated $r=y \oplus z$.

Damgård and Lunemann [12] proved that Blum's coin-flipping protocol is quantum-secure, assuming a quantum-secure commitment scheme. This protocol generates one random coin, and we will need to flip logarithmic many random bits. A simple way of achieving this is by sequential repetition, but more effectively it is possible to extend the analysis of Damgård and Lunemann and show that parallel repetition of Blum's protocol logarithmic many times remains quantum-secure.

\section{A.6 Concatenated Steane codes}

The last topic to be discussed in this section concerns the existence of quantum error correcting codes having certain properties that are important to the functioning of our zero-knowledge proof system for QMA. There are multiple choices of codes that satisfy our requirements, but in the interest of simplicity we will describe just one specific family of codes in this category.

These codes are based on the 7-qubit Steane code [44], in which one qubit is encoded into 7 qubits by the following action on standard basis states:

$$
|0\rangle \mapsto \frac{1}{\sqrt{8}} \sum_{x \in \mathcal{D}_{7}^{0}}|x\rangle \quad \text { and } \quad|1\rangle \mapsto \frac{1}{\sqrt{8}} \sum_{x \in \mathcal{D}_{7}^{1}}|x\rangle,
$$

where

$$
\begin{aligned}
& \mathcal{D}_{7}^{0}=\{0000000,0001111,0110011,0111100,1010101,1011010,1100110,1101001\} \\
& \mathcal{D}_{7}^{1}=\{0010110,0011001,0100101,0101010,1000011,1001100,1110000,1111111\} .
\end{aligned}
$$

It is the case that $\mathcal{D}_{7}^{0}$ is a $[7,4]$-Hamming code, while

$$
\mathcal{D}_{7}=\mathcal{D}_{7}^{0} \cup \mathcal{D}_{7}^{1}
$$

is the dual code to $\mathcal{D}_{7}^{0}$ (i.e., it is the code consisting of all binary strings of length 7 whose inner product with any codeword in $\mathcal{D}_{7}^{0}$ is even). This is an example of a CSS code [41], and it is capable of correcting single-qubit errors. The standard error-correcting procedure, which we do not actually need in this paper, is to first reversibly correct errors in the standard basis, with respect to the code $\mathcal{D}_{7}$, and then to do the same with respect to the diagonal basis. The 7-qubit Clifford circuit depicted in Figure 12 encodes one qubit into 7 with respect to this code, assuming 6 qubits in the $|0\rangle$ state are made available. 


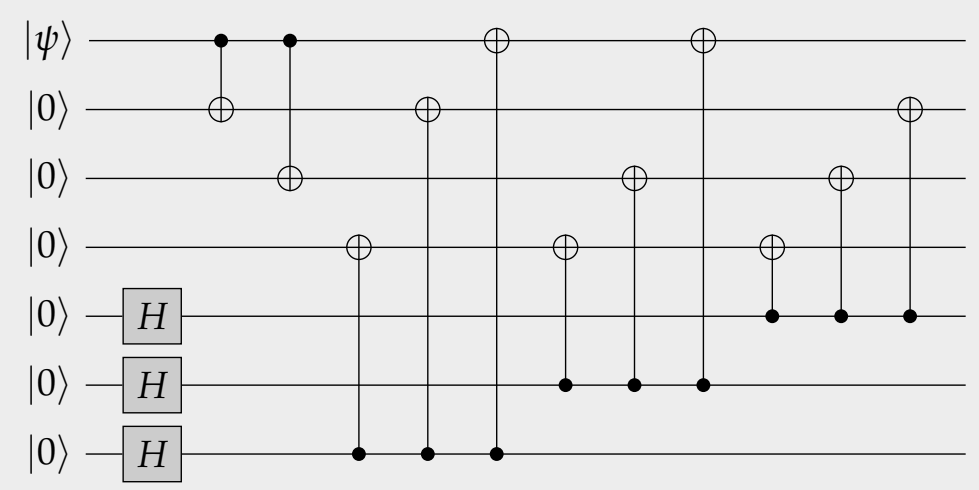

Figure 12: A Clifford circuit encoder for the 7-qubit Steane code. Hereafter we will write $U_{7}$ to refer to the unitary operator on 7 qubits described by this circuit.

One of the properties of the 7-qubit Steane code that is important from the viewpoint of this paper is that it admits a transversal application of Clifford operations, in the sense that is explained in Figure 13.

Note that by concatenating the 7-qubit Steane code with itself, one obtains a code having similar properties to the 7-qubit code, and in addition having a large minimum distance for the underlying code. More specifically, suppose that $N=7^{t}$ for $t$ being an even positive integer. (We take $t$ to be even for convenience, as this eliminates the entry-wise complex conjugation on Clifford operations encountered in the discussion of their transversal application.) By concatenating the 7-qubit Steane code to itself $t$ times, one obtains a quantum error-correcting code in which one qubit is encoded into $N$ qubits in the following way:

$$
|0\rangle \mapsto \frac{1}{\sqrt{8^{t}}} \sum_{x \in \mathcal{D}_{N}^{0}}|x\rangle \quad \text { and } \quad|1\rangle \mapsto \frac{1}{\sqrt{8^{t}}} \sum_{x \in \mathcal{D}_{N}^{1}}|x\rangle
$$

where $\mathcal{D}_{N}^{0}, \mathcal{D}_{N}^{1} \subseteq\{0,1\}^{N}$ are related in a way that generalizes the case $N=7$. In particular, $\mathcal{D}_{N}^{0}$ is a binary linear code having $8^{t}$ elements, and whose dual code takes the form

$$
\mathcal{D}_{N}=\mathcal{D}_{N}^{0} \cup \mathcal{D}_{N}^{1}
$$

for $\mathcal{D}_{N}^{1} \subseteq\{0,1\}^{N}$ being a coset of $\mathcal{D}_{N}^{0}$.

As a quantum error correcting code, the $t$-fold concatenation of the 7-qubit Steane code inherits the properties of the 7-qubit Steane code mentioned above. A Clifford circuit $U_{N}$ acting on $N$ qubits, $N-1$ of which are to be initialized in the $|0\rangle$ state, performs the encoding. This circuit is obtained by creating a tree from multiple copies of the circuit $U_{7}$ in the natural way. The code allows for Clifford operations to be applied transversally.

An added feature of the concatenated versions of the 7-qubit Steane code is that it corrects more errors than the ordinary 7-qubit code. In particular, we will make use of the fact that the code $\mathcal{D}_{N}$, for $N=7^{t}$, has minimum Hamming weight $3^{t}$ for a nonzero code word. This allows one to obtain a polynomial-length code for any polynomial lower-bound on the minimum nonzero Hamming weight of a code word. 

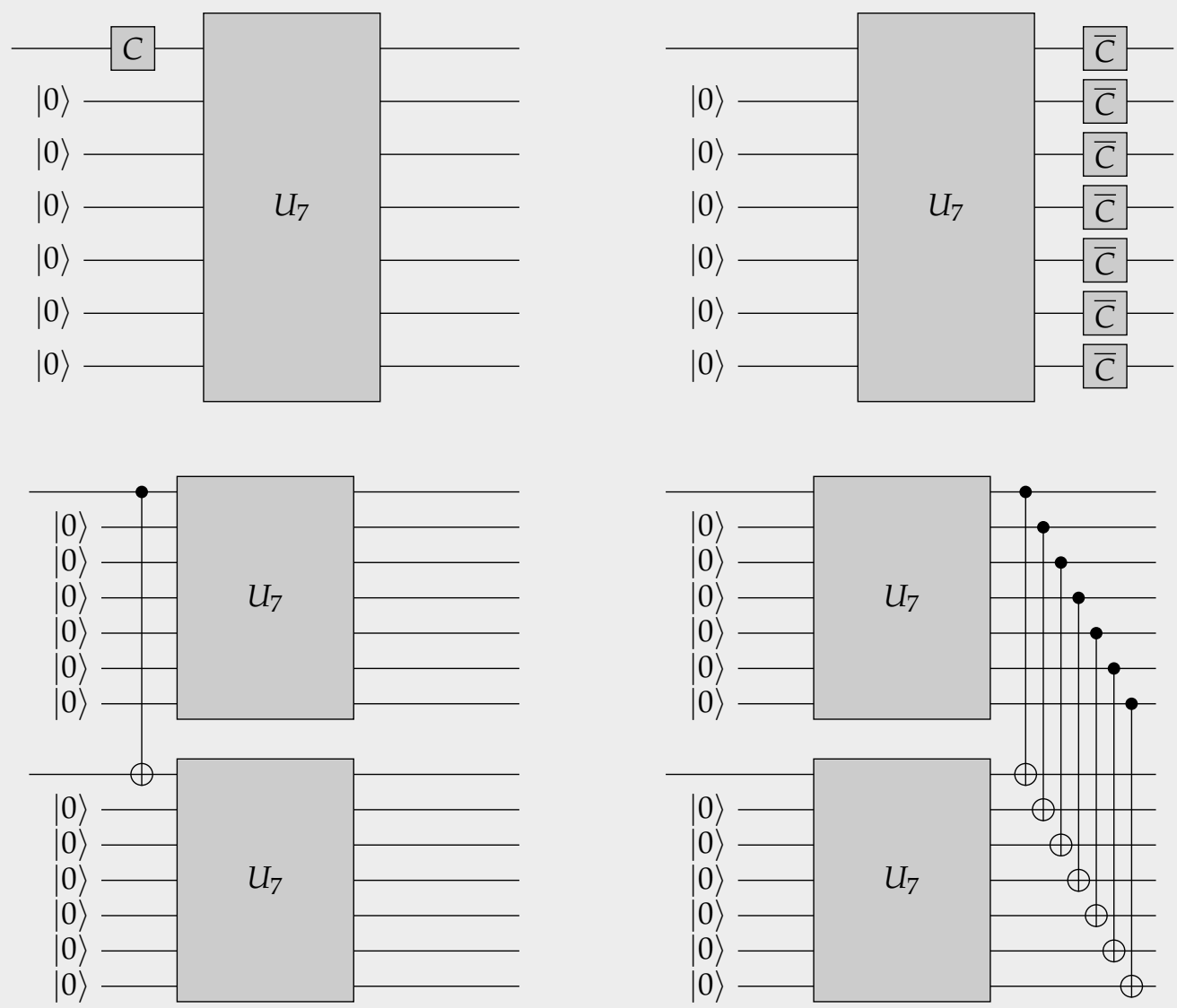

Figure 13: The 7-qubit Steane code allows for the transversal application of Clifford operations. That is, the circuits on the left are equivalent to the corresponding circuits on the right. In general, the application of any Clifford operation on $k$ qubits prior to being encoded is equivalent to the entry-wise complex conjugate of that Clifford operation being applied 7 times to the $7 k$ qubits that encode the original $k$ qubits.

\section{References}

[1] AdCOCK, M., AND CLEVE, R. A quantum Goldreich-Levin theorem with cryptographic applications. In Proceedings of the 19th International Symposium on Theoretical Aspects of Computer Science, vol. 2285 of Lecture Notes in Computer Science. Springer-Verlag, 2002, pp. 323-334.

[2] Aharonov, D., BEN-Or, M., AND EBAn, E. Interactive proofs for quantum computations. In Innovations in Computer Science (2010), pp. 453-469.

[3] Aharonov, D., Kitaev, A., And Nisan, N. Quantum circuits with mixed states. In Proceedings of the 30th Annual ACM Symposium on Theory of Computing (1998), pp. 20-30.

[4] Ambainis, A., Mosca, M., TAPP, A., AND DE Wolf, R. Private quantum channels. In Proceedings of the 41st Annual IEEE Symposium on Foundations of Computer Science (2000), pp. 547553. 
[5] Barnum, H., Crépeau, C., Gottesman, D., Smith, A., and Tapp, A. Authentication of quantum messages. In Proceedings of the 43th Annual IEEE Symposium on Foundations of Computer Science (2002), pp. 449-458.

[6] Ben-Or, M., Crépeau, C., Gottesman, D., Hassidim, A., And Smith, A. Secure multiparty quantum computation with (only) a strict honest majority. In Proceedings of the 47th Annual IEEE Symposium on Foundations of Computer Science (2006), pp. 249-260.

[7] Ben-Or, M., Goldreich, O., Goldwasser, S., HÅstad, J., Kilian, J., Micali, S., And ROGAWAY, P. Everything provable is provable in zero-knowledge. In Advances in CryptologyCRYPTO 1988 (1990), vol. 403 of Lecture Notes in Computer Science, Springer-Verlag, pp. 37-56.

[8] BLum, M. Coin flipping by telephone a protocol for solving impossible problems. ACM SIGACT News 15, 1 (1983), 23-27.

[9] BRAVYI, S. Efficient algorithms for a quantum analogue of 2-SAT. Contemporary Mathematics 536 (2011), 33-48.

[10] Broadbent, A., Gutoski, G., And Stebila, D. Quantum one-time programs. In Advances in Cryptology - CRYPTO 2013 (2013), vol. 8043 of Lecture Notes in Computer Science, Springer, pp. 344-360.

[11] DAMGÅRD, I., FEHR, S., AND SALVAIL, L. Zero-knowledge proofs and string commitments withstanding quantum attacks. In Advances in Cryptology - CRYPTO 2004 (2004), vol. 3152 of Lecture Notes in Computer Science, Springer, pp. 254-272.

[12] DAMGÅRD, I., AND LunEMANN, C. Quantum-secure coin-flipping and applications. In Advances in Cryptology - ASIACRYPT 2009 (2009), vol. 5912 of Lecture Notes in Computer Science, Springer, pp. 52-69.

[13] Dupuis, F., Nielsen, J. B., AND SAlvail, L. Secure two-party quantum evaluation of unitaries against specious adversaries. In Advances in Cryptology-CRYPTO 2010 (2010), vol. 6223 of Lecture Notes in Computer Science, Springer, pp. 685-706.

[14] Dupuis, F., Nielsen, J. B., AND SAlvail, L. Actively secure two-party evaluation of any quantum operation. In Advances in Cryptology - CRYPTO 2012 (2012), vol. 7417 of Lecture Notes in Computer Science, Springer, pp. 794-811.

[15] FeIGE, U., AND SHAmiR, A. Zero knowledge proofs of knowledge in two rounds. In Advances in Cryptology - CRYPTO 1989 (1990), vol. 435 of Lecture Notes in Computer Science, SpringerVerlag, pp. 526-544.

[16] FuCHS, C. A., AND PERES, A. Quantum-state disturbance versus information gain: Uncertainty relations for quantum information. Physical Review A 53, 4 (1996), 2038.

[17] Goldreich, O. Foundations of Cryptography I: Basic Tools. Cambridge University Press, 2001.

[18] Goldreich, O. Foundations of Cryptography II: Basic Applications. Cambridge University Press, 2004.

[19] Goldreich, O., AND KAHAN, A. How to construct constant-round zero-knowledge proof systems for NP. Journal of Cryptology 9, 3 (1996), 167-189. 
[20] Goldreich, O., Micali, S., And Wigderson, A. How to play ANY mental game. In Proceedings of the 19th Annual ACM Symposium on Theory of Computing (1987), pp. 218-229.

[21] Goldreich, O., Micali, S., And Wigderson, A. Proofs that yield nothing but their validity or all languages in NP have zero-knowledge proof systems. Journal of the ACM 38, 3 (1991), 690-728.

[22] Goldreich, O., AND OREn, Y. Definitions and properties of zero-knowledge proof systems. Journal of Cryptology 7, 1 (1994), 1-32.

[23] GOldwasser, S., MiCAli, S., AND RACKOFF, C. The knowledge complexity of interactive proof systems. SIAM Journal on Computing 18, 1 (1989), 186-208.

[24] Goldwasser, S., AND SiPSER, M. Private coins versus public coins in interactive proof systems. In Proceedings of the 18th Annual ACM Symposium on Theory of Computing (1986), pp. 59-68.

[25] Gosset, D., AND NAGAJ, D. Quantum 3-SAT is QMA $_{1}$-complete. In Proceedings of the 54th Annual IEEE Symposium on Foundations of Computer Science (2013), pp. 756-765.

[26] Gottesman, D. The Heisenberg representation of quantum computers. In Group 22: Proceedings of the 22nd International Colloquium on Group Theoretical Methods in Physics (1998), pp. 3243.

[27] Hallgren, S., Kolla, A., Sen, P., AND Zhang, S. Making classical honest verifier zero knowledge protocols secure against quantum attacks. In Proceedings of the 35th International Colloquium on Automata, Languages and Programming, Part II (2008), vol. 5126 of Lecture Notes in Computer Science, Springer-Verlag, pp. 592-603.

[28] Hallgren, S., SMith, A., AND SONG, F. Classical cryptographic protocols in a quantum world. International Journal of Quantum Information 13, 04 (2015), 1550028.

[29] HÅstad, J., Impagliazzo, R., Levin, L. A., And Luby, M. A pseudorandom generator from any one-way function. SIAM Journal on Computing 28, 4 (1999), 1364-1396.

[30] Impagliazzo, R. A personal view of average-case complexity. In Proceedings of 10th Annual IEEE Structure in Complexity Theory Conference (1995), pp. 134-147.

[31] Kempe, J., KitAeV, A., And RegeV, O. The complexity of the local Hamiltonian problem. SIAM Journal on Computing 35, 5 (2006), 1070-1097.

[32] KeMPE, J., AND ReGEV, O. 3-local Hamiltonian is QMA-complete. Quantum Information and Computation 3, 3 (2003), 258-264.

[33] KITAEV, A. Y. Quantum computations: algorithms and error correction. Russian Mathematical Surveys 52, 6 (1997), 1191-1249.

[34] Kitaev, A. Y., Shen, A. H., AND Vyalyi, M. N. Classical and Quantum Computation, vol. 47 of Graduate Studies in Mathematics. American Mathematical Society, 2002.

[35] LIU, Y.-K. Consistency of local density matrices is QMA-complete. In Proceedings of the 9th International Workshop on Approximation Algorithms for Combinatorial Optimization Problems, APPROX 2006 and 10th International Workshop on Randomization and Computation, RANDOM 2006, vol. 4110 of Lecture Notes in Computer Science. Springer-Verlag, 2006, pp. 438-449. 
[36] Lunemann, C., AND Nielsen, J. B. Fully simulatable quantum-secure coin-flipping and applications. In Progress in Cryptology - AFRICACRYPT 2011 (2011), vol. 6737 of Lecture Notes in Computer Science, Springer-Verlag, pp. 21-40.

[37] Marriott, C., AND Watrous, J. Quantum Arthur-Merlin games. Computational Complexity 14,2 (2005), 122-152.

[38] Morimae, T., Hayashi, M., Nishimura, H., And FujiI, K. Quantum Merlin-Arthur with Clifford Arthur. Quantum Information and Computation 15 (2015), 1420-1430.

[39] Morimae, T., NAgaJ, D., AND SCHUCH, N. Quantum proofs can be verified using only single-qubit measurements. Physical Review A 93, 2 (2016), 022326.

[40] NAOR, M. Bit commitment using pseudorandomness. Journal of Cryptology 4, 2 (1991), 151158.

[41] Nielsen, M., AND ChuANG, I. Quantum Computation and Quantum Information. Cambridge University Press, 2000.

[42] SHOR, P. W. Polynomial-time algorithms for prime factorization and discrete logarithms on a quantum computer. SIAM Journal on Computing 26, 5 (1997), 1484-1509.

[43] SONG, F. A note on quantum security for post-quantum cryptography. In Proceedings of the 6th International Workshop on Post-Quantum Cryptography, vol. 8772 of Lecture Notes in Computer Science. Springer, 2014, pp. 246-265.

[44] Steane, A. Multi-particle interference and quantum error correction. Proceedings of the Royal Society A 452 (1996), 2551-2577.

[45] VAN DE GRAAF, J. Towards a formal definition of security for quantum protocols. PhD thesis, Université de Montréal, 1997.

[46] Watrous, J. Limits on the power of quantum statistical zero-knowledge. In Proceedings of the 43rd Annual IEEE Symposium on Foundations of Computer Science (2002), pp. 459-468.

[47] WATROUS, J. PSPACE has constant-round quantum interactive proof systems. Theoretical Computer Science 292, 3 (2003), 575-588.

[48] WATrous, J. Quantum computational complexity. In Encyclopedia of complexity and systems science. Springer, 2009, pp. 7174-7201.

[49] WAtrous, J. Zero-knowledge against quantum attacks. SIAM Journal on Computing 39, 1 (2009), 25-58.

[50] WAtrous, J. An introduction to quantum information and quantum circuits. ACM SIGACT News 42, 2 (2011), 52-67.

[51] Wootters, W. K., And ZureK, W. H. A single quantum cannot be cloned. Nature 299 (1982), 802-803.

[52] ZHANDRY, M. How to construct quantum random functions. In Proceedings of the 53rd Annual IEEE Symposium on Foundations of Computer Science (2012), pp. 679-687. 